\title{
Rigidity of quasiconformal maps on Carnot groups
}

\author{
Xiangdong Xie*
}

\begin{abstract}
We show that quasiconformal maps on many Carnot groups must be biLipschitz. In particular, this is the case for 2-step Carnot groups with reducible first layer. These results have implications for the rigidity of quasiisometries between negatively curved solvable Lie groups.
\end{abstract}

Keywords. rigidity, Carnot group, quasisymmetric map, biLipschitz. Mathematics Subject Classification (2000). 22E25, 30L10, 53C17.

\section{Introduction}

In this paper we establish rigidity results for quasiconformal maps between Carnot groups. We show that quasiconformal maps on many Carnot groups must be biLipschitz.

Let $\mathcal{N}=V_{1} \oplus \cdots \oplus V_{r}$ be a Carnot Lie algebra. For each $t>0$, the dilation $\lambda_{t}: \mathcal{N} \rightarrow \mathcal{N}$ is defined by $\lambda_{t}(v)=t^{i} v$ for $v \in V_{i}$. An isomorphism $A: \mathcal{N} \rightarrow \mathcal{N}$ is called a graded isomorphism if $A$ commutes with the dilations $\lambda_{t}$ for all $t>0$; that is, $A \circ \lambda_{t}=\lambda_{t} \circ A$ for all $t>0$. Let $\operatorname{Aut}_{g}(\mathcal{N})$ be the group of graded isomorphisms of $\mathcal{N}$. We say $V_{1}$ is reducible (or the first layer of $\mathcal{N}$ is reducible) if there is a non-trivial proper linear subspace $W_{1} \subset V_{1}$ such that $A\left(W_{1}\right)=W_{1}$ for every $A \in \operatorname{Aut}_{g}(\mathcal{N})$.

Let $K \geq 1$ and $C>0$. A bijection $F: X \rightarrow Y$ between two metric spaces is called a $(K, C)$-quasi-similarity if

$$
\frac{C}{K} d(x, y) \leq d(F(x), F(y)) \leq C K d(x, y)
$$

for all $x, y \in X$.

Clearly a map is a quasi-similarity if and only if it is biLipschitz. The point here is that often there is control on $K$ but not on $C$. In this case, the notion of quasi-similarity provides more information about the distortion.

We say that a Carnot group $N$ is quasisymmetrically rigid if every $\eta$-quasisymmetric map is a $(K, C)$-quasi-similarity, where $K$ is a constant depending only on $\eta$. See Section 2.3 for the definition of quasisymmetric map.

Here is our first result:

\footnotetext{
${ }^{*}$ Partially supported by NSF grant DMS-1265735.
} 
Theorem 1.1. Let $N$ be a 2-step Carnot group. If the first layer of its Lie algebra is reducible, then $N$ is quasisymmetrically rigid.

Of course, for many Carnot Lie algebras, the first layer is not reducible. On the other hand, every Carnot algebra is a subalgebra of some Carnot algebra with reducible first layer. See Section 4.2 for some constructions.

For general Carnot groups, we have the following:

Theorem 1.2. Suppose $W_{1} \subset V_{1}$ is a non-trivial proper subspace that is invariant under the action of $\operatorname{Aut}_{g}(\mathcal{N})$. If there is some $X \in V_{1} \backslash W_{1}$ such that $\left[X, W_{1}\right] \subset\left[W_{1}, W_{1}\right]$, then $N$ is quasisymmetrically rigid.

The following result is very useful for induction argument:

Theorem 1.3. Suppose $W_{1} \subset V_{1}$ is a non-trivial proper subspace that is invariant under the action of $A u t_{g}(\mathcal{N})$. Let $W$ be the connected subgroup of $N$ with Lie algebra $\left\langle W_{1}\right\rangle$. If $W$ is quasisymmetrically rigid, then so is $N$.

The only Carnot groups known to admit non-biLipschitz quasiconformal maps are the Heisenberg groups $[\mathrm{B}$. We conjecture that the Heisenberg groups are the only exceptions.

The results in this paper extend previous results by the author for reducible Carnot groups [X] and model Filiform groups [X2]. In a forthcoming paper [X1] the author proves that even most non-rigid Carnot groups are quasisymmetrically rigid.

The first rigidity theorem about quasiconformal maps of Carnot groups is due to Pansu. He proved that $([\mathrm{P}])$ every quasiconformal map of the quarternionic Heisenberg group is a composition of left translations and graded automorphisms. In particular, the space of quasiconformal maps is finite dimensional. Our results are of somehow different nature. Our results imply that many Carnot groups with rank at most one are quasisymmetrically rigid. It will be shown in [X1] that the space of biLipschitz maps of Carnot groups with rank at most one is infinite dimensional. See Section 4.1 for definition of rank and Section 6] for examples of quasisymmetrically rigid Carnot groups with rank at most one.

The results in this paper have implications for the large scale geometry of negatively curved homogeneous manifolds. Each Carnot group arises as the (one point complement of) ideal boundary of some negatively curved homogeneous manifold $[\mathrm{H}]$. Furthermore, each quasiisometry of the negatively curved homogeneous manifold associated to a quasisymmetrically rigid Carnot group is a rough isometry, that is, it must preserve the distance up to an additive constant.

In Section 2 we recall basic definitions and facts about Carnot groups. In Section 3 we prove Theorems 1.2 and 1.3. In Section 4 we explain how to find invariant subspaces and introduce constructions that produce Carnot algebras with reducible first layer (so that the Theorems in this paper can be applied). In Section 5 we prove Theorem 1.1. Finally in Section [6] we apply our results to 3 explicit examples.

Acknowledgment. This work was completed while the author was attending the workshop "Interactions between analysis and geometry" at IPAM, University of California at Los Angeles from March to June 2013. I would like to thank IPAM for financial support, excellent working conditions and conducive atmosphere. I also would like to thank Tullia Dymarz and David Freeman for discussions about Carnot groups. 


\section{Preliminaries}

In this Section we collect definitions and results that shall be needed later. We first recall the basic definitions related to Carnot groups in Subsection 2.1. Then we review the BCH formula (Subsection 2.2), the definition of quasisymmetric maps (Subsection 2.3) and Pansu differentiability theorem (Subsection 2.4).

\subsection{Carnot algebras and Carnot groups}

A Carnot Lie algebra is a finite dimensional Lie algebra $\mathcal{G}$ over $\mathbb{R}$ together with a direct sum decomposition $\mathcal{G}=V_{1} \oplus V_{2} \oplus \cdots \oplus V_{r}$ of non-trivial vector subspaces such that $\left[V_{1}, V_{i}\right]=$ $V_{i+1}$ for all $1 \leq i \leq r$, where we set $V_{r+1}=\{0\}$. The integer $r$ is called the degree of nilpotency of $\mathcal{G}$. Every Carnot algebra $\mathcal{G}=V_{1} \oplus V_{2} \oplus \cdots \oplus V_{r}$ admits a one-parameter family of automorphisms $\lambda_{t}: \mathcal{G} \rightarrow \mathcal{G}, t \in(0, \infty)$, where $\lambda_{t}(x)=t^{i} x$ for $x \in V_{i}$. Let $\mathcal{G}=V_{1} \oplus V_{2} \oplus \cdots \oplus V_{r}$ and $\mathcal{G}^{\prime}=V_{1}^{\prime} \oplus V_{2}^{\prime} \oplus \cdots \oplus V_{s}^{\prime}$ be two Carnot algebras. A Lie algebra homomorphism $\phi: \mathcal{G} \rightarrow \mathcal{G}^{\prime}$ is graded if $\phi$ commutes with $\lambda_{t}$ for all $t>0$; that is, if $\phi \circ \lambda_{t}=\lambda_{t} \circ \phi$. We observe that $\phi\left(V_{i}\right) \subset V_{i}^{\prime}$ for all $1 \leq i \leq r$.

A simply connected nilpotent Lie group is a Carnot group if its Lie algebra is a Carnot algebra. Let $G$ be a Carnot group with Lie algebra $\mathcal{G}=V_{1} \oplus \cdots \oplus V_{r}$. The subspace $V_{1}$ defines a left invariant distribution $H G \subset T G$ on $G$. We fix a left invariant inner product on $H G$. An absolutely continuous curve $\gamma$ in $G$ whose velocity vector $\gamma^{\prime}(t)$ is contained in $H_{\gamma(t)} G$ for a.e. $t$ is called a horizontal curve. By Chow's theorem ([BR, Theorem 2.4]), any two points of $G$ can be connected by horizontal curves. Let $p, q \in G$, the Carnot metric $d_{c}(p, q)$ between them is defined as the infimum of length of horizontal curves that join $p$ and $q$.

Since the inner product on $H G$ is left invariant, the Carnot metric on $G$ is also left invariant. Different choices of inner product on $H G$ result in Carnot metrics that are biLipschitz equivalent. The Hausdorff dimension of $G$ with respect to a Carnot metric is given by $\sum_{i=1}^{r} i \cdot \operatorname{dim}\left(V_{i}\right)$.

Recall that, for a simply connected nilpotent Lie group $G$ with Lie algebra $\mathcal{G}$, the exponential map exp : $\mathcal{G} \rightarrow G$ is a diffeomorphism. Under this identification the Lesbegue measure on $\mathcal{G}$ is a Haar measure on $G$. Furthermore, the exponential map induces a oneto-one correspondence between Lie subalgebras of $\mathcal{G}$ and connected Lie subgroups of $G$.

Let $G$ be a Carnot group with Lie algebra $\mathcal{G}=V_{1} \oplus \cdots \oplus V_{r}$. Since $\lambda_{t}: \mathcal{G} \rightarrow \mathcal{G}(t>0)$ is a Lie algebra automorphism and $G$ is simply connected, there is a unique Lie group automorphism $\Lambda_{t}: G \rightarrow G$ whose differential at the identity is $\lambda_{t}$. For each $t>0, \Lambda_{t}$ is a similarity with respect to the Carnot metric: $d\left(\Lambda_{t}(p), \Lambda_{t}(q)\right)=t d(p, q)$ for any two points $p, q \in G$. A Lie group homomorphism $f: G \rightarrow G^{\prime}$ between two Carnot groups is a graded homomorphism if it commutes with $\Lambda_{t}$ for all $t>0$; that is, if $f \circ \Lambda_{t}=\Lambda_{t} \circ f$. Notice that, a Lie group homomorphism $f: G \rightarrow G^{\prime}$ between two Carnot groups is graded if and only if the corresponding Lie algebra homomorphism is graded. 


\subsection{The Baker-Campbell-Hausdorff formula}

Let $G$ be a simply connected nilpotent Lie group with Lie algebra $\mathcal{G}$. The exponential map $\exp : \mathcal{G} \rightarrow G$ is a diffeomorphism. One can then pull back the group operation from $G$ to get a group stucture on $\mathcal{G}$. This group structure can be described by the Baker-CampbellHausdorff formula (BCH formula in short), which expresses the product $X * Y(X, Y \in \mathcal{G})$ in terms of the iterated Lie brackets of $X$ and $Y$. The group operation in $G$ will be denoted by $\cdot$. The pull-back group operation $*$ on $\mathcal{G}$ is defined as follows. For $X, Y \in \mathcal{G}$, define

$$
X * Y=\exp ^{-1}(\exp X \cdot \exp Y)
$$

Then the first a few terms of the $\mathrm{BCH}$ formula ( $\mathrm{CG}$, page 11) is given by:

$$
X * Y=X+Y+\frac{1}{2}[X, Y]+\frac{1}{12}[X,[X, Y]]-\frac{1}{12}[Y,[X, Y]]+\cdots .
$$

Next we define homogeneous distances on Carnot groups. With the help of BCH formula, it is often more convenient to work with homogeneous distances than with Carnot metrics. Let $G$ be a Carnot group with Lie algebra $\mathcal{G}=V_{1} \oplus V_{2} \oplus \cdots \oplus V_{r}$. Write $x \in \mathcal{G}$ as $x=x_{1}+\cdots+x_{r}$ with $x_{i} \in V_{i}$. Fix a norm $|\cdot|$ on each layer. Define a norm $\|\cdot\|$ on $\mathcal{G}$ by:

$$
\|x\|=\sum_{i=1}^{r}\left|x_{i}\right|^{\frac{1}{i}} .
$$

Now define a homogeneous distance on $G=\mathcal{G}$ by: $d(g, h)=\|(-g) * h\|$. An important fact is that $d$ and $d_{c}$ are biLipschitz equivalent. That is, there is a constant $C \geq 1$ such that $d(p, q) / C \leq d_{c}(p, q) \leq C \cdot d(p, q)$ for all $p, q \in G$. It is often possible to calculate or estimate $d$ by using the $\mathrm{BCH}$ formula. Since we are only concerned with quasiconformal maps and biLipschitz maps, it does not matter whether we use $d$ or $d_{c}$.

\subsection{Quasisymmetric maps}

Here we recall the definition of quasisymmetric map.

Let $\eta:[0, \infty) \rightarrow[0, \infty)$ be a homeomorphism. A homeomorphism $F: X \rightarrow Y$ between two metric spaces is $\eta$-quasisymmetric if for all distinct triples $x, y, z \in X$, we have

$$
\frac{d(F(x), F(y))}{d(F(x), F(z))} \leq \eta\left(\frac{d(x, y)}{d(x, z)}\right)
$$

If $F: X \rightarrow Y$ is an $\eta$-quasisymmetry, then $F^{-1}: Y \rightarrow X$ is an $\eta_{1}$-quasisymmetry, where $\eta_{1}(t)=\left(\eta^{-1}\left(t^{-1}\right)\right)^{-1}$. See [V], Theorem 6.3. A homeomorphism between metric spaces is quasisymmetric if it is $\eta$-quasisymmetric for some $\eta$.

We remark that quasisymmetric homeomorphisms between general metric spaces are quasiconformal. In the case of Carnot groups (and more generally Loewner spaces), a homeomorphism is quasisymmetric if and only if it is quasiconformal, see [HK].

The main result in [BKR] says that a quasiconformal map between two proper, locally Ahlfors $Q$-regular $(Q>1)$ metric spaces is absolutely continuous on almost every curve. This result applies to quasiconformal maps $F: G \rightarrow G$ on Carnot groups. 
Pansu [P] proved that a quasisymmetric map $F: G_{1} \rightarrow G_{2}$ between two Carnot groups is absolutely continuous: a measurable set $A \subset G_{1}$ has measure 0 if and only if $F(A)$ has measure 0 .

Let $g:\left(X_{1}, \rho_{1}\right) \rightarrow\left(X_{2}, \rho_{2}\right)$ be a homeomorphism between two metric spaces. We define for every $x \in X_{1}$ and $r>0$,

$$
\begin{aligned}
L_{g}(x, r) & =\sup \left\{\rho_{2}\left(g(x), g\left(x^{\prime}\right)\right): \rho_{1}\left(x, x^{\prime}\right) \leq r\right\}, \\
l_{g}(x, r) & =\inf \left\{\rho_{2}\left(g(x), g\left(x^{\prime}\right)\right): \rho_{1}\left(x, x^{\prime}\right) \geq r\right\},
\end{aligned}
$$

and set

$$
L_{g}(x)=\limsup _{r \rightarrow 0} \frac{L_{g}(x, r)}{r}, \quad l_{g}(x)=\liminf _{r \rightarrow 0} \frac{l_{g}(x, r)}{r} .
$$

Then

$$
L_{g^{-1}}(g(x))=\frac{1}{l_{g}(x)} \text { and } l_{g^{-1}}(g(x))=\frac{1}{L_{g}(x)}
$$

for any $x \in X_{1}$. If $g$ is an $\eta$-quasisymmetry, then

$$
L_{g}(x, r) \leq \eta(1) l_{g}(x, r)
$$

for all $x \in X_{1}$ and $r>0$. Hence if in addition

$$
\lim _{r \rightarrow 0} \frac{L_{g}(x, r)}{r} \text { or } \lim _{r \rightarrow 0} \frac{l_{g}(x, r)}{r}
$$

exists, then

$$
0 \leq l_{g}(x) \leq L_{g}(x) \leq \eta(1) l_{g}(x) \leq \infty .
$$

\subsection{Pansu differentiability theorem}

First the definition:

Definition 2.3. Let $G$ and $G^{\prime}$ be two Carnot groups endowed with Carnot metrics, and $U \subset G, U^{\prime} \subset G^{\prime}$ open subsets. A map $F: U \rightarrow U^{\prime}$ is Pansu differentiable at $x \in U$ if there exists a graded homomorphism $L: G \rightarrow G^{\prime}$ such that

$$
\lim _{y \rightarrow x} \frac{d\left(F(x)^{-1} * F(y), L\left(x^{-1} * y\right)\right)}{d(x, y)}=0 .
$$

In this case, the graded homomorphism $L: G \rightarrow G^{\prime}$ is called the Pansu differential of $F$ at $x$, and is denoted by $d F(x)$.

We have the following chain rule for Pansu differentials:

Lemma 2.1. (Lemma 3.7 in [CC]) Suppose $F_{1}: U_{1} \rightarrow U_{2}$ is Pansu differentiable at $p$, and $F_{2}: U_{2} \rightarrow U_{3}$ is Pansu differentiable at $F_{1}(p)$. Then $F_{2} \circ F_{1}$ is Pansu differentiable at $p$ and $d\left(F_{2} \circ F_{1}\right)(p)=d F_{2}\left(F_{1}(p)\right) \circ d F_{1}(p)$. 
Notice that the Pansu differential of the identity map $U_{1} \rightarrow U_{1}$ is the identity isomorphism. Hence if $F: U_{1} \rightarrow U_{2}$ is bijective, $F$ is Pansu differentiable at $p \in U_{1}$ and $F^{-1}$ is Pansu differentiable at $F(p)$, then $d F^{-1}(F(p))=(d F(p))^{-1}$.

The following result (except the terminology) is due to Pansu $[\mathrm{P}]$.

Theorem 2.2. Let $G, G^{\prime}$ be Carnot groups, and $U \subset G, U^{\prime} \subset G^{\prime}$ open subsets. Let $F: U \rightarrow U^{\prime}$ be a quasiconformal map. Then $F$ is a.e. Pansu differentiable. Furthermore, at a.e. $x \in U$, the Pansu differential $d F(x): G \rightarrow G^{\prime}$ is a graded isomorphism.

In Theorem 2.2 and the proofs below, "a. e." is with respect to the Lesbegue measure on $\mathcal{G}=G$.

\section{Quasiconformal implies biLipschitz}

In this Section, we prove Theorem 1.2 and Theorem 1.3. They provide very useful sufficient conditions for a Carnot group to be quasisymmetrically rigid.

We shall need the following result.

Proposition 3.1. (Proposition 3.4 in [X] ) Let $G$ and $G^{\prime}$ be two Carnot groups with Lie algebras $\mathcal{G}=V_{1} \oplus \cdots \oplus V_{m}$ and $\mathcal{G}^{\prime}=V_{1}^{\prime} \oplus \cdots \oplus V_{n}^{\prime}$ respectively. Let $W_{1} \subset V_{1}, W_{1}^{\prime} \subset V_{1}^{\prime}$ be subspaces. Denote by $\mathcal{W} \subset \mathcal{G}$ and $\mathcal{W}^{\prime} \subset \mathcal{G}^{\prime}$ respectively the Lie subalgebras generated by $W_{1}$ and $W_{1}^{\prime}$. Let $W \subset G$ and $W^{\prime} \subset G^{\prime}$ respectively be the connected Lie subgroups of $G$ and $G^{\prime}$ corresponding to $\mathcal{W}$ and $\mathcal{W}^{\prime}$. Let $F: G \rightarrow G^{\prime}$ be a quasisymmetric homeomorphism. If $d F(x)\left(W_{1}\right) \subset W_{1}^{\prime}$ for a.e. $x \in G$, then $F$ sends left cosets of $W$ into left cosets of $W^{\prime}$.

Let $N$ be a Carnot group with Lie algebra $\mathcal{N}=V_{1} \oplus \cdots \oplus V_{r}$. For any linear subspace $W_{1} \subset V_{1}$, let $\mathcal{W}$ be the subalgebra of $\mathcal{N}$ generated by $W_{1}$. Denote by $W$ the connected subgroup of $N$ with Lie algebra $\mathcal{W}$. Notice that $\mathcal{W}$ is also a Carnot algebra and can be written as $\mathcal{W}=W_{1} \oplus \cdots \oplus W_{r}$. In general, there is some integer $1 \leq s \leq r$ such that $W_{s} \neq 0$ and $W_{j}=0$ for $j>s$.

The following result is very useful for induction argument.

Theorem 3.2. Let $W_{1} \subset V_{1}$ be a non-trivial proper subspace that is invariant under the action of $\operatorname{Aut}_{g}(\mathcal{N})$. Suppose $W$ has the following property: every $\eta$-quasisymmetric map $G: W \rightarrow W$ is a $(K, C)$-quasi-similarity, where $K$ depends only on $\eta$. Then $N$ has the same property (possibly with a different constant $K^{\prime}$ depending only on $\eta$ ).

Proof. The proof is similar to the arguments in the proofs of Lemma 3.9 and Lemma 3.10 in X2]. We include it here mainly for completeness. Let $F: N \rightarrow N$ be an $\eta$-quassymmetric map. By Proposition [3.1, $F$ sends left cosets of $W$ to left cosets of $W$. For each left coset $L$ of $W$, the restriction $\left.F\right|_{L}: L \rightarrow F(L)$ is also an $\eta$-quasisymmetric map. Let $L_{1}=n_{1} * W, L_{2}=n_{2} * W$ be two left cosets of $W$. By assumption, $\left.F\right|_{L_{i}}$ is a $\left(K, C_{i}\right)$ quasi-similarity, where $K \geq 1$ and $C_{i}>0$. Here $K$ depends only on $\eta$. We shall first show that $C_{1} / 2 K^{2} \leq C_{2} \leq 2 K^{2} C_{1}$. Suppose $C_{2}>2 K^{2} C_{1}$. We will get a contradiction from this. Fix some $w \in W_{1}$ with $|w|=1$ and consider the image of the two horizontal lines $n_{1} * t w$ 
$(t \in \mathbb{R}), n_{2} * t w(t \in \mathbb{R})$ under $F$. First of all, by using BCH formula it is easy to show that $d\left(n_{1} * t w, n_{2} * t w\right)$ is bounded from above by a sublinear function of $t$ as $t \rightarrow \infty$. Since $d\left(n_{1}, n_{1} * t w\right)=t$, we have

$$
\frac{d\left(n_{1} * t w, n_{2} * t w\right)}{d\left(n_{1} * t w, n_{1}\right)} \rightarrow 0
$$

as $t \rightarrow \infty$. The quasisymmetric condition now implies

$$
\frac{d\left(F\left(n_{1} * t w\right), F\left(n_{2} * t w\right)\right)}{d\left(F\left(n_{1} * t w\right), F\left(n_{1}\right)\right)} \rightarrow 0 .
$$

On the other hand, since $\left.F\right|_{L_{i}}$ is a $\left(K, C_{i}\right)$ quasi-similarity, we have

$$
d\left(F\left(n_{2}\right), F\left(n_{2} * t w\right)\right) \geq C_{2} / K \cdot d\left(n_{2}, n_{2} * t w\right)>2 K C_{1} \cdot t
$$

and

$$
d\left(F\left(n_{1}\right), F\left(n_{1} * t w\right)\right) \leq K C_{1} \cdot d\left(n_{1}, n_{1} * t w\right)=K C_{1} \cdot t .
$$

It follows that

$$
\begin{aligned}
\frac{d\left(F\left(n_{1} * t w\right), F\left(n_{2} * t w\right)\right)}{d\left(F\left(n_{1} * t w\right), F\left(n_{1}\right)\right)} & \geq \frac{d\left(F\left(n_{2} * t w\right), F\left(n_{2}\right)\right)-d\left(F\left(n_{2}\right), F\left(n_{1}\right)\right)-d\left(F\left(n_{1}\right), F\left(n_{1} * t w\right)\right)}{K C_{1} t} \\
& \geq \frac{2 K C_{1} t-d\left(F\left(n_{2}\right), F\left(n_{1}\right)\right)-K C_{1} t}{K C_{1} t} \rightarrow 1 .
\end{aligned}
$$

The contradiction shows $C_{2} \leq 2 K^{2} C_{1}$. Similarly, $C_{1} / 2 K^{2} \leq C_{2}$.

We next show that $F$ is a quasi-similarity. Fix any left coset $L$ of $W$. Then, as indicated above, $\left.F\right|_{L}$ is a $\left(K, C_{0}\right)$-quasi-similarity for some $C_{0}>0$. Let $p, q \in N$ be arbitrary points. Then there are left cosets $L_{1}, L_{2}$ of $W$ with $p \in L_{1}, q \in L_{2}$. By the preceding paragraph, $\left.F\right|_{L_{1}}$ is a $\left(2 K^{3}, C_{0}\right)$-quasi-similarity. Pick $q^{\prime} \in L_{1}$ with $d\left(p, q^{\prime}\right)=d(p, q)$. The quasisymmetry condition implies that

$$
d(F(p), F(q)) \leq \eta(1) \cdot d\left(F(p), F\left(q^{\prime}\right)\right) \leq \eta(1) \cdot 2 K^{3} \cdot C_{0} \cdot d\left(p, q^{\prime}\right)=2 K^{3} \eta(1) \cdot C_{0} \cdot d(p, q) .
$$

Now the same argument applied to $F^{-1}$ finishes the proof.

Lemma 3.3. Suppose $W_{1} \subset V_{1}$ is a non-trivial proper subspace that is invariant under the action of $\operatorname{Aut}_{g}(\mathcal{N})$. If $\mathcal{W}=<W_{1}>$ is an ideal in $\mathcal{N}$, then every $\eta$-quasisymmetric map $F: N \rightarrow N$ is a $(K, C)$-quasi-similarity, where $K$ depends only on $\eta$.

Proof. Since $\mathcal{W}$ is an ideal in $\mathcal{N}$, the subgroup $W$ corresponding to $\mathcal{W}$ is a connected normal subgroup of $N$. By Proposition 3.1, $F$ maps cosets of $W$ to cosets of $W$. Since $W$ is normal in $N$, any two cosets $n_{1} * W, n_{2} * W$ of $W$ are "parallel" in the following sense: for any $p \in n_{1} * W$, we have $d\left(p, n_{2} * W\right)=d\left(n_{1} * W, n_{2} * W\right)$. Furthermore, the quotient $N / W$ is a Carnot group with Lie algebra $V_{1} / W_{1} \oplus \cdots \oplus V_{r} / W_{r}$, and the distance between $n_{1} * W$ and $n_{2} * W$ in $N$ equals the distance between the two points $n_{1} W, n_{2} W$ in $N / W$. In particular, the metrics on the quotient and each coset are geodesic. Now the argument in [SX] implies that $F$ is a $(K, C)$-quasi-similarity, where $K$ depends only on $\eta$. 
Theorem 3.4. Suppose $W_{1} \subset V_{1}$ is a non-trivial proper subspace that is invariant under the action of $\operatorname{Aut}_{g}(\mathcal{N})$. Let $N\left(W_{1}\right)=\left\{v \in V_{1}:\left[v, W_{1}\right] \subset \mathcal{W}\right\}$. If $N\left(W_{1}\right) \neq W_{1}$, then every $\eta$-quasisymmetric map $F: N \rightarrow N$ is a $(K, C)$-quasi-similarity, where $K$ depends only on $\eta$.

Proof. Notice that $N\left(W_{1}\right)$ is also invariant under the action of $\operatorname{Aut}_{g}(\mathcal{N})$. Let $N_{2} \subset N$ be the connected subgroup of $N$ with Lie algebra $\left\langle N\left(W_{1}\right)\right\rangle$. Let $F: N \rightarrow N$ be an $\eta$-quasisymmetric map. Proposition 3.1 implies that $F$ sends left cosets of $N_{2}$ to left cosets of $N_{2}$ and also sends left cosets of $W$ to left cosets of $W$. Clearly, for each left coset $L$ of $N_{2}$, the restriction $\left.F\right|_{L}: L \rightarrow F(L)$ is also $\eta$-quasisymmetric. Notice that $\mathcal{W}$ is an ideal of $<N\left(W_{1}\right)>$. Since $N\left(W_{1}\right) \neq W_{1}$, (the proof of) Lemma 3.3 yields that the restriction $\left.F\right|_{L}$ is a $(K, C)$-quasi-similarity, where the constant $K$ depends only on $\eta$. Now the Theorem follows from Theorem 3.2 .

\section{Invariant subspaces}

In this Section we first explain how to find invariant subspaces, then introduce some constructions that produce Carnot algebras with reducible first layer.

\subsection{Rank and invariant subspaces}

In this Subsection we define the rank of elements in a Lie algebra, and explain how to use rank to find invariant subspaces. The notion of rank appeared in [O]. There is a characterization of non-rigid Carnot groups in terms of rank [OW].

Let $f: X \rightarrow Y$ be a linear map between two vector spaces. The $\operatorname{rank} \operatorname{rank}(f)$ of $f$ is defined to be the dimension of the image $f(X)$ of $f$. Recall that for any positive integer $m$, $f$ induces a linear map $\wedge^{m} f: \wedge^{m} X \rightarrow \wedge^{m} Y$ between the $m$-fold exterior products.

Lemma 4.1. Let $k$ be a non-negative integer and $f: X \rightarrow Y$ be a linear map. Then $\operatorname{rank}(f) \leq k$ if and only if $\wedge^{k+1} f=0$.

Proof. First assume $\operatorname{rank}(f) \leq k$. Then $f(X)$ has dimension at most $k$. Hence for any $x_{1}, \cdots, x_{k+1} \in X$, we have

$$
\wedge^{k+1} f\left(x_{1} \wedge \cdots \wedge x_{k+1}\right)=f\left(x_{1}\right) \wedge \cdots \wedge f\left(x_{k+1}\right)=0 .
$$

Conversely, assume $\operatorname{rank}(f) \geq k+1$. Then there are vectors $x_{1}, \cdots, x_{k+1} \in X$ such that $f\left(x_{1}\right), \cdots, f\left(x_{k+1}\right)$ are linearly independent. Hence

$$
\wedge^{k+1} f\left(x_{1} \wedge \cdots \wedge x_{k+1}\right)=f\left(x_{1}\right) \wedge \cdots \wedge f\left(x_{k+1}\right) \neq 0 .
$$


For an element $x \in \mathcal{N}$ in a Lie algebra, let $\operatorname{rank}(x)$ be the rank of the linear transformation $\operatorname{ad}(x): \mathcal{N} \rightarrow \mathcal{N}, \operatorname{ad}(x)(y)=[x, y]$. In other words, $\operatorname{rank}(x)$ is the dimension of the image of $a d(x)$. For any isomorphism $A: \mathcal{N}_{1} \rightarrow \mathcal{N}_{2}$ of Lie algebras, we have $\operatorname{rank}(x)=\operatorname{rank}(A(x))$ for any $x \in \mathcal{N}_{1}$. The rank of a Lie algebra $\mathcal{N}$ is defined by

$$
\operatorname{rank}(\mathcal{N})=\min \{\operatorname{rank}(x): 0 \neq x \in \mathcal{N}\}
$$

For any $\lambda \neq 0$ and any $x \in \mathcal{N}$, we clearly have $\operatorname{rank}(\lambda x)=\operatorname{rank}(x)$. Hence the rank induces a function $R: P \mathcal{N} \rightarrow \mathbb{Z}$ on the projective space $P \mathcal{N}:$

$$
R([x])=\operatorname{rank}(x) \text { for } 0 \neq x \in \mathcal{N} \text {. }
$$

Set $A_{k}=\{[x] \in P \mathcal{N}: \operatorname{rank}(x) \leq k\}$. Clearly $A_{k} \subset A_{k+1}$ for all $k$.

Let $\left\{e_{1}, \cdots, e_{n}\right\}$ be a basis of a Lie algebra $\mathcal{N}$. Then any element $x \in \mathcal{N}$ can be written as: $x=x_{1} e_{1}+\cdots+x_{n} e_{n}\left(x_{i} \in \mathbb{R}\right)$. Then $\operatorname{ad}(x)=x_{1} a d\left(e_{1}\right)+\cdots+x_{n} a d\left(e_{n}\right)$. It follows that

$$
\wedge^{m} a d(x)=\sum_{i_{1}, \cdots, i_{m}} x_{i_{1}} \cdots x_{i_{m}} a d\left(e_{i_{1}}\right) \wedge \cdots \wedge a d\left(e_{i_{m}}\right)
$$

is a linear combination of the linear maps $a d\left(e_{i_{1}}\right) \wedge \cdots \wedge a d\left(e_{i_{m}}\right)$ and the coefficients are polynomials in $x_{1}, \cdots, x_{n}$. Now it is easy to see by using Lemma 4.1 that $A_{k} \subset P \mathcal{N}$ is an algebraic variety.

Now let $\mathcal{N}=V_{1} \oplus \cdots \oplus V_{r}$ be a Carnot algebra. Define

$$
r_{1}(\mathcal{N})=\min \left\{\operatorname{rank}(x): 0 \neq x \in V_{1}\right\} .
$$

The quantity $r_{1}(\mathcal{N})$ will be called the rank of $\mathcal{N}$ and of the corresponding Carnot group $N$. Let $W_{1} \subset V_{1}$ be the subspace of $V_{1}$ spanned by elements $x \in V_{1}$ satisfying $\operatorname{rank}(x)=r_{1}(\mathcal{N})$. Since graded isomorphisms preserve the first layer $V_{1}$, we see that $W_{1}$ is a non-trivial subspace of $V_{1}$ invariant under the action of $\operatorname{Aut}_{g}(\mathcal{N})$. Very often $W_{1}$ is a proper subspace of $V_{1}$. For example, this is the case for most non-rigid Carnot groups [X1]. Similarly, for $1 \leq i \leq r-1$, define

$$
r_{1, i}(\mathcal{N})=\min \left\{\operatorname{rank}\left(\left.a d(x)\right|_{V_{i}}\right): 0 \neq x \in V_{1}\right\}
$$

Let $W_{1, i} \subset V_{1}$ be the subspace of $V_{1}$ spanned by elements $x \in V_{1}$ satisfying $\operatorname{rank}\left(\left.\operatorname{ad}(x)\right|_{V_{i}}\right)=$ $r_{1, i}(\mathcal{N})$. Then $W_{1, i}$ is also a non-trivial subspace of $V_{1}$ invariant under the action of $\operatorname{Aut}_{g}(\mathcal{N})$.

Since $\left\{A_{k}\right\}$ is a nested sequence of algebraic varieties, the best chance to obtain proper invariant subspace is to consider elements of minimal rank. This is why we defined the subspaces $W_{1}$ and $W_{1, i}$.

Recall that a Carnot group is called rigid if the space of smooth contact maps is finite dimensional. Ottazzi $([\mathrm{O}])$ showed that if $N$ has rank at most one, then $N$ is non-rigid. Furthermore, Ottazzi and Warhurst ([OW]) proved that a Carnot group is non-rigid if and only if the complexification $\mathcal{N} \otimes \mathbb{C}$ of its Lie algebra has rank at most one (as a complex Lie algebra). 


\subsection{Product constructions}

In this Subsection we introduce a few product type constructions for Carnot algebras and Carnot groups. They produce Carnot algebras with proper invariant subspaces.

Direct Product. The basic product construction is the direct product of two Lie algebras. We will use the direct sum notation. Let $\mathcal{N}=V_{1} \oplus \cdots \oplus V_{m}$ and $\mathcal{N}^{\prime}=V_{1}^{\prime} \oplus \cdots \oplus V_{n}^{\prime}$ be two Carnot algebras. We may assume $m \geq n$. We will write $\mathcal{N}^{\prime}=V_{1}^{\prime} \oplus \cdots \oplus V_{m}^{\prime}$ with $V_{i}^{\prime}=\{0\}$ for $i>n$. Then $\mathcal{N} \oplus \mathcal{N}^{\prime}=\left(V_{1} \oplus V_{1}^{\prime}\right) \oplus \cdots \oplus\left(V_{m} \oplus V_{m}^{\prime}\right)$ is also a Carnot algebra. Notice that if $x_{1} \in V_{1}$ and $x_{1}^{\prime} \in V_{1}^{\prime}$, then $\operatorname{rank}\left(x_{1}+x_{1}^{\prime}\right)=\operatorname{rank}\left(x_{1}\right)+\operatorname{rank}\left(x_{1}^{\prime}\right)$. It follows that, if $r_{1}(\mathcal{N}) \neq r_{1}\left(\mathcal{N}^{\prime}\right)$, then the subspace of $V_{1} \oplus V_{1}^{\prime}$ spanned by elements of minimal rank is a non-trivial proper subspace invariant under the action of $\operatorname{Aut}_{g}\left(\mathcal{N} \oplus \mathcal{N}^{\prime}\right)$.

Central Product. For any ideal $\mathcal{I} \subset \mathcal{N} \oplus \mathcal{N}^{\prime}$, the quotient $\left(\mathcal{N} \oplus \mathcal{N}^{\prime}\right) / \mathcal{I}$ is a Lie algebra. In general, this quotient is not a Carnot algebra. An ideal $\mathcal{I} \subset \mathcal{N} \oplus \mathcal{N}^{\prime}$ is called a graded ideal if $\mathcal{I}=\left[\mathcal{I} \cap\left(V_{1} \oplus V_{1}^{\prime}\right)\right] \oplus \cdots \oplus\left[\mathcal{I} \cap\left(V_{m} \oplus V_{m}^{\prime}\right)\right]$. If $\mathcal{I} \subset \mathcal{N} \oplus \mathcal{N}^{\prime}$ is a graded ideal, then the quotient $\left(\mathcal{N} \oplus \mathcal{N}^{\prime}\right) / \mathcal{I}$ is also a Carnot algebra: $\left(\mathcal{N} \oplus \mathcal{N}^{\prime}\right) / \mathcal{I}=V_{1}^{\prime \prime} \oplus \cdots \oplus V_{m}^{\prime \prime}$, where

$$
V_{i}^{\prime \prime}=\left(V_{i} \oplus V_{i}^{\prime}\right) /\left[\left(V_{i} \oplus V_{i}^{\prime}\right) \cap \mathcal{I}\right] .
$$

Let $\mathcal{I} \subset V_{m} \oplus V_{m}^{\prime}$ be any linear subspace. Since $V_{m} \oplus V_{m}^{\prime}$ lies in the center of $\mathcal{N} \oplus \mathcal{N}^{\prime}$, we see that $\mathcal{I}$ is a graded ideal of $\mathcal{N} \oplus \mathcal{N}^{\prime}$. So the associated quotient is a Carnot algebra. A particular case is the following. Let $W \subset V_{m}, W^{\prime} \subset V_{m}^{\prime}$ be linear subspaces and $f: W \rightarrow W^{\prime}$ a linear isomorphism. Then $\mathcal{I}=\{f(w)-w \mid w \in W\}$ is a graded ideal of $\mathcal{N} \oplus \mathcal{N}^{\prime}$. The associated quotient is called a central product of the two Carnot algebras $\mathcal{N}$ and $\mathcal{N}^{\prime}$.

Let $\overline{\mathcal{N}}$ be a central product of $\mathcal{N}$ and $\mathcal{N}^{\prime}$. Notice that the compositions $\mathcal{N} \hookrightarrow \mathcal{N} \oplus \mathcal{N}^{\prime} \rightarrow$ $\overline{\mathcal{N}}, \mathcal{N}^{\prime} \hookrightarrow \mathcal{N} \oplus \mathcal{N}^{\prime} \rightarrow \overline{\mathcal{N}}$ are injective, and $\left[V_{1}, \mathcal{N}^{\prime}\right]=0,\left[V_{1}^{\prime}, \mathcal{N}\right]=0$. It follows that each $x_{1} \in V_{1}$ has the same rank as an element in $\mathcal{N}$ and an element in $\overline{\mathcal{N}}$. Similarly for $x_{1}^{\prime} \in V_{1}^{\prime}$. Furthermore, if $x_{1} \in V_{1}$ and $x_{1}^{\prime} \in V_{1}^{\prime}$, then $\operatorname{rank}\left(x_{1}+x_{1}^{\prime}\right) \geq \max \left\{\operatorname{rank}\left(x_{1}\right), \operatorname{rank}\left(x_{1}^{\prime}\right)\right\}$. Consequently, if $r_{1}(\mathcal{N}) \neq r_{1}\left(\mathcal{N}^{\prime}\right)$, then the subspace of $V_{1} \oplus V_{1}^{\prime} \subset \overline{\mathcal{N}}$ spanned by elements of minimal rank is a non-trivial proper subspace invariant under the action of $\operatorname{Aut}_{g}(\overline{\mathcal{N}})$. We have observed that both $\mathcal{N}$ and $\mathcal{N}^{\prime}$ are isomorphic to their images in the central product.

Given an $m$-step Carnot algebra $\mathcal{N}$ with $m \geq 2$, one can always find another $m$-step Carnot algebra $\mathcal{N}^{\prime}$ with $r_{1}\left(\mathcal{N}^{\prime}\right)>r_{1}(\mathcal{N})$. For instance, one may choose an $m$-step free nilpotent Lie algebra on sufficiently many generators. Hence, by the above discussion, $\mathcal{N}$ always embeds in a Carnot algebra with a reducible first layer.

Level One Product. Now let $\mathcal{N}=V_{1} \oplus V_{2}$ and $\mathcal{N}^{\prime}=V_{1}^{\prime} \oplus V_{2}^{\prime}$ be 2-step Carnot algebras. Let $X_{0} \in V_{1} \backslash\{0\}$ and $X_{0}^{\prime} \in V_{1}^{\prime} \backslash\{0\}$. Let $U_{1} \subset V_{1}$ be a subspace complementary to $\mathbb{R} X_{0}$ and $U_{1}^{\prime} \subset V_{1}^{\prime}$ a subspace complementary to $\mathbb{R} X_{0}^{\prime}$. We define a new 2-step Carnot algebra $\overline{\mathcal{N}}=\bar{V}_{1} \oplus \bar{V}_{2}$ as follows. The first layer $\bar{V}_{1}=U_{1} \oplus U_{1}^{\prime} \oplus \mathbb{R} X$, where $\mathbb{R} X$ is a 1-dimensional vector space with basis vector $X$. The second layer $\bar{V}_{2}=V_{2} \oplus V_{2}^{\prime}$. The bracket on $\overline{\mathcal{N}}$ is defined as follows. For any $u_{1}, u_{2} \in U_{1}, u_{1}^{\prime}, u_{2}^{\prime} \in U_{1}^{\prime}, a, b \in \mathbb{R}$, define

$$
\begin{aligned}
& {\left[u_{1}+u_{1}^{\prime}+a X, u_{2}+u_{2}^{\prime}+b X\right] } \\
= & {\left[u_{1}, u_{2}\right]+b \cdot\left[u_{1}, X_{0}\right]+\left[u_{1}^{\prime}, u_{2}^{\prime}\right]+b \cdot\left[u_{1}^{\prime}, X_{0}^{\prime}\right]+a \cdot\left[X_{0}, u_{2}\right]+a \cdot\left[X_{0}^{\prime}, u_{2}^{\prime}\right] . }
\end{aligned}
$$

We call $\overline{\mathcal{N}}$ a level one product of $\mathcal{N}$ and $\mathcal{N}^{\prime}$. It depends on the choices of $X_{0}, X_{0}^{\prime}, U_{1}, U_{1}^{\prime}$. We notice that both $\mathcal{N}$ and $\mathcal{N}^{\prime}$ are isomorphic to their images in the level one product. 
For the following Lemma, notice that we can always choose $X_{0}, X_{0}^{\prime}$ and $U_{1}, U_{1}^{\prime}$ so that $U_{1}$ and $U_{1}^{\prime}$ contain elements of minimal rank.

Lemma 4.2. Let $\overline{\mathcal{N}}$ be a level one product of $\mathcal{N}$ and $\mathcal{N}^{\prime}$ defind as above. Suppose there are $\tilde{u}_{1} \in U_{1}$ and $\tilde{u}_{1}^{\prime} \in U_{1}^{\prime}$ such that $\operatorname{rank}\left(\tilde{u}_{1}\right)=r_{1}(\mathcal{N})$ and $\operatorname{rank}\left(\tilde{u}_{1}^{\prime}\right)=r_{1}\left(\mathcal{N}^{\prime}\right)$. If $r_{1}(\mathcal{N}) \geq 1$ and $r_{1}\left(\mathcal{N}^{\prime}\right) \geq 1$, then $\overline{\mathcal{N}}$ has a reducible first layer.

Proof. To avoid confusion, if $v \in \overline{V_{1}}$, we use $\bar{r}(v)$ to denote the rank of $v$ in the Lie algebra $\overline{\mathcal{N}}$. We still use $\operatorname{rank}(x)$ to denote rank of elements $x$ in the Lie algebras $\mathcal{N}$ and $\mathcal{N}^{\prime}$. The definition of the Lie bracket in $\overline{\mathcal{N}}$ shows that for any $u_{1} \in U_{1}, u_{1}^{\prime} \in U_{1}^{\prime}$, we have $\left[u_{1}, \overline{V_{1}}\right]=$ $\left[u_{1}, V_{1}\right]$ and $\left[u_{1}^{\prime}, \overline{V_{1}}\right]=\left[u_{1}^{\prime}, V_{1}^{\prime}\right]$. This implies $\bar{r}\left(u_{1}\right)=\operatorname{rank}\left(u_{1}\right)$ and $\bar{r}\left(u_{1}^{\prime}\right)=\operatorname{rank}\left(u_{1}^{\prime}\right)$. By the assumption, there are $\tilde{u}_{1} \in U_{1}$ and $\tilde{u}_{1}^{\prime} \in U_{1}^{\prime}$ such that $\bar{r}\left(\tilde{u}_{1}\right)=r_{1}(\mathcal{N})$ and $\bar{r}\left(\tilde{u}_{1}^{\prime}\right)=r_{1}\left(\mathcal{N}^{\prime}\right)$. We next show that if $v \in \overline{V_{1}} \backslash\left(U_{1} \oplus U_{1}^{\prime}\right)$, then $\bar{r}(v) \geq r_{1}(\mathcal{N})+r_{1}\left(\mathcal{N}^{\prime}\right)$. Since we assume $r_{1}(\mathcal{N}) \geq 1$ and $r_{1}\left(\mathcal{N}^{\prime}\right) \geq 1$, this implies that all elements of minimal rank are contained in $U_{1} \oplus U_{1}^{\prime}$, and the Lemma follows.

Let $v \in \overline{V_{1}} \backslash\left(U_{1} \oplus U_{1}^{\prime}\right)$. Write $v$ as $v=u_{1}+u_{1}^{\prime}+a X$ with $u_{1} \in U_{1}, u_{1}^{\prime} \in U_{1}^{\prime}$ and $a \neq 0$. We may assume $a=1$. So $v=u_{1}+u_{1}^{\prime}+X$. Then

$$
\left[v, \overline{V_{1}}\right]=\left\{\left[u_{1}+X_{0}, u_{2}\right]+b\left[u_{1}, X_{0}\right]+\left[u_{1}^{\prime}+X_{0}^{\prime}, u_{2}^{\prime}\right]+b\left[u_{1}^{\prime}, X_{0}^{\prime}\right]: u_{2} \in U_{1}, u_{2}^{\prime} \in U_{1}^{\prime}, b \in \mathbb{R}\right\} .
$$

Let $P: \overline{V_{2}}=V_{2} \oplus V_{2}^{\prime} \rightarrow V_{2}$ be the projection. Then

$$
\begin{aligned}
P\left(\left[v, \overline{V_{1}}\right]\right) & =\left\{\left[u_{1}+X_{0}, u_{2}\right]+b\left[u_{1}, X_{0}\right]: u_{2} \in U_{1}, b \in \mathbb{R}\right\} \\
& =\left\{\left[u_{1}+X_{0}, u_{2}\right]+\left[u_{1}+X_{0}, b X_{0}\right]: u_{2} \in U_{1}, b \in \mathbb{R}\right\} \\
& =\left\{\left[u_{1}+X_{0}, u_{2}+b X_{0}\right]: u_{2} \in U_{1}, b \in \mathbb{R}\right\} \\
& =\left[u_{1}+X_{0}, V_{1}\right] .
\end{aligned}
$$

Hence $\operatorname{dim}\left(P\left(\left[v, \overline{V_{1}}\right]\right)\right)=\operatorname{rank}\left(u_{1}+X_{0}\right) \geq r_{1}(\mathcal{N})$. On the other hand,

$$
\begin{aligned}
& {\left[v, \overline{V_{1}}\right] \cap P^{-1}(0) } \\
= & \left\{\left[u_{1}^{\prime}+X_{0}^{\prime}, u_{2}^{\prime}\right]+b\left[u_{1}^{\prime}, X_{0}^{\prime}\right]:\left[u_{1}+X_{0}, u_{2}\right]+b\left[u_{1}, X_{0}\right]=0, u_{2} \in U_{1}, u_{2}^{\prime} \in U_{1}^{\prime}, b \in \mathbb{R}\right\} \\
= & \left\{\left[u_{1}^{\prime}+X_{0}^{\prime}, u_{2}^{\prime}+b X_{0}^{\prime}\right]:\left[u_{1}+X_{0}, u_{2}\right]+b\left[u_{1}, X_{0}\right]=0, u_{2} \in U_{1}, u_{2}^{\prime} \in U_{1}^{\prime}, b \in \mathbb{R}\right\} .
\end{aligned}
$$

Notice that there is no restriction on $u_{2}^{\prime}$. Also, for any $b \in \mathbb{R}$, the equality $\left[u_{1}+X_{0}, u_{2}\right]+$ $b\left[u_{1}, X_{0}\right]=0$ holds for $u_{2}=b u_{1}$. Hence $\left[v, \overline{V_{1}}\right] \cap P^{-1}(0)=\left[u_{1}^{\prime}+X_{0}^{\prime}, V_{1}^{\prime}\right]$. It follows that

$$
\operatorname{dim}\left(\left[v, \overline{V_{1}}\right] \cap P^{-1}(0)\right)=\operatorname{rank}\left(u_{1}^{\prime}+X_{0}^{\prime}\right) \geq r_{1}\left(\mathcal{N}^{\prime}\right)
$$

and so

$$
\bar{r}(v)=\operatorname{dim}\left(\left[v, \overline{V_{1}}\right]\right)=\operatorname{dim}\left(P\left(\left[v, \overline{V_{1}}\right]\right)\right)+\operatorname{dim}\left(\left[v, \overline{V_{1}}\right] \cap P^{-1}(0)\right) \geq r_{1}(\mathcal{N})+r_{1}\left(\mathcal{N}^{\prime}\right) .
$$




\section{QC maps on 2-step Carnot groups}

In this Section we prove Theorem 1.1 ,

Throughout this Section, $N$ will be a 2-step Carnot group with Lie algebra $\mathcal{N}=V_{1} \oplus V_{2}, W_{1} \subset V_{1}$ a non-trivial proper subspace invariant under the action of $\operatorname{Aut}_{g}(\mathcal{N})$ on $V_{1}$, and $F: N \rightarrow N$ an $\eta$-quasisymmetric map.

Let $\mathcal{W}$ be the subalgebra of $\mathcal{N}$ generated by $W_{1}$, and $W$ the connected subgroup of $N$ with Lie algebra $\mathcal{W}$. Notice that $\mathcal{W}$ is also a Carnot algebra and can be written as $\mathcal{W}=W_{1} \oplus W_{2}$. We may have $W_{2}=0$. This happens if and only if $W_{1}$ is abelian; that is, $\left[W_{1}, W_{1}\right]=0$. Let $\tilde{W}_{1} \subset V_{1}$ be a subspace complementary to $W_{1}$, and $\tilde{W}_{2} \subset V_{2}$ be a subspace complementary to $W_{2}$. Then we have $V_{1}=W_{1} \oplus \tilde{W}_{1}$ and $V_{2}=W_{2} \oplus \tilde{W}_{2}$. We denote by $P_{2}: V_{2} \rightarrow W_{2}$ and $\tilde{P}_{2}: V_{2} \rightarrow \tilde{W}_{2}$ respectively the projections with respect to the direct sum decomposition $V_{2}=W_{2} \oplus \tilde{W}_{2}$. That is, if $w=w_{2}+\tilde{w}_{2}$ with $w_{2} \in W_{2}$ and $\tilde{w}_{2} \in \tilde{W}_{2}$, then $P_{2}(w)=w_{2}$ and $\tilde{P}_{2}(w)=\tilde{w}_{2}$.

Theorem 1.1 follows from Lemma 3.3 in the case when $W_{2}=V_{2}$. From now on we shall assume $W_{2} \neq V_{2}$. We fix inner products on $V_{1}$ and $V_{2}$ such that $W_{i}$ and $\tilde{W}_{i}$ are perpendicular. Then there exists some constant $A \geq 1$ such that $|[v, w]| \leq A \cdot|v| \cdot|w|$ for all $v, w \in V_{1}$. Fix some $e \in \tilde{W}_{2}$ with $|e|=1$.

We recall that, if $F: X \rightarrow Y$ is $\eta$-quasisymmetric, then $F^{-1}: Y \rightarrow X$ is $\eta_{1^{-}}$ quasisymmetric with $\eta_{1}(t)=\left(\eta^{-1}\left(t^{-1}\right)\right)^{-1}$. Without loss of generality we may assume $\eta(1) \geq 1$. It follows that $\eta_{1}(1) \geq 1$. These inequalities will be implicitly used throughout the paper.

Lemma 5.1. Suppose $W_{2} \neq V_{2}$. Let $L$ be a left coset of $W$. Suppose $p, q \in L$ are such that $l_{F}(p)>C_{1} \cdot L_{F}(q)$ with $C_{1}=200 A \eta_{1}(1)$. Write $q=p *\left(u_{1}+u_{2}\right)$ with $u_{1} \in W_{1}$ and $u_{2} \in W_{2}$. Then for any $s=p *\left(t u_{1}+w_{2}^{\prime}\right)$ with $|t| \geq 1$ and $w_{2}^{\prime} \in W_{2}$, we have

$$
L_{F}(s) \leq \frac{2\left(\eta_{1}(1)\right)^{2}}{\sqrt{|t|}} \cdot L_{F}(q) .
$$

Proof. Set $p^{\prime}=F(p), q^{\prime}=F(q)$ and $L^{\prime}=F(L)$. The assumption and (2.1) imply $l_{F^{-1}}\left(q^{\prime}\right)>$ $C_{1} \cdot L_{F^{-1}}\left(p^{\prime}\right)$. Let $\left\{r_{j}\right\}$ be an arbitrary sequence of positive reals such that $r_{j} \rightarrow 0$. Then

$$
\liminf \frac{l_{F^{-1}}\left(q^{\prime}, r_{j}\right)}{r_{j}} \geq C_{1} \cdot \limsup \frac{L_{F^{-1}}\left(p^{\prime}, r_{j}\right)}{r_{j}}
$$

Fix some $e \in \tilde{W}_{2}$ with $|e|=1$. We shall look at the image of $r_{j}^{2} e * L^{\prime}$ under $F^{-1}$.

Denote $\bar{p}_{j}=r_{j}^{2} e * p^{\prime}$ and $\bar{q}_{j}=r_{j}^{2} e * q^{\prime}$. Since $d\left(p^{\prime}, \bar{p}_{j}\right)=d\left(q^{\prime}, \bar{q}_{j}\right)=r_{j}$, we have

$$
\frac{d\left(F^{-1}\left(\bar{q}_{j}\right), q\right)}{r_{j}} \geq \frac{l_{F^{-1}}\left(q^{\prime}, r_{j}\right)}{r_{j}}
$$

and

$$
\frac{d\left(F^{-1}\left(\bar{p}_{j}\right), p\right)}{r_{j}} \leq \frac{L_{F^{-1}}\left(p^{\prime}, r_{j}\right)}{r_{j}} .
$$


Let $p_{j}, q_{j}$ be points on $F^{-1}\left(r_{j}^{2} e * L^{\prime}\right)$ nearest to $p$ and $q$ respectively. Since $W_{2}$ and $\tilde{W}_{2}$ are perpendicular, we have $d\left(p^{\prime}, \bar{p}_{j}\right)=d\left(p^{\prime}, r_{j}^{2} e * L^{\prime}\right)=r_{j}$. In particular, $d\left(p^{\prime}, \bar{p}_{j}\right) \leq d\left(p^{\prime}, F\left(p_{j}\right)\right)$. Now the quasisymmetry condition of $F^{-1}$ implies $d\left(p, F^{-1}\left(\bar{p}_{j}\right)\right) \leq \eta_{1}(1) d\left(p, p_{j}\right)$. Similarly we have $d\left(q, F^{-1}\left(\bar{q}_{j}\right)\right) \leq \eta_{1}(1) d\left(q, q_{j}\right)$. It follows that

$$
\frac{d\left(q_{j}, q\right)}{r_{j}} \geq \frac{1}{\eta_{1}(1)} \cdot \frac{d\left(F^{-1}\left(\bar{q}_{j}\right), q\right)}{r_{j}} \geq \frac{1}{\eta_{1}(1)} \cdot \frac{l_{F^{-1}}\left(q^{\prime}, r_{j}\right)}{r_{j}} .
$$

The choice of $p_{j}$ implies

$$
\frac{d\left(p_{j}, p\right)}{r_{j}} \leq \frac{d\left(F^{-1}\left(\bar{p}_{j}\right), p\right)}{r_{j}} \leq \frac{L_{F^{-1}}\left(p^{\prime}, r_{j}\right)}{r_{j}}
$$

It follows from (5.2), (5.1) and (5.3) that

$$
\begin{aligned}
\liminf \frac{d\left(q_{j}, q\right)}{r_{j}} \geq \liminf \frac{1}{\eta_{1}(1)} \cdot \frac{l_{F^{-1}}\left(q^{\prime}, r_{j}\right)}{r_{j}} & \geq \frac{C_{1}}{\eta_{1}(1)} \cdot \limsup \frac{L_{F^{-1}}\left(p^{\prime}, r_{j}\right)}{r_{j}} \\
& \geq \frac{C_{1}}{\eta_{1}(1)} \cdot \limsup \frac{d\left(p_{j}, p\right)}{r_{j}}
\end{aligned}
$$

Now the choice of $C_{1}$ implies that

$$
d\left(p, p_{j}\right) \leq \frac{1}{101 A} d\left(q, q_{j}\right)
$$

for all sufficiently large $j$.

Next we shall look at $d\left(p, p_{j}\right)$ and $d\left(q, q_{j}\right)$.

Since $q, p$ lie on the same left coset, we can write $q=p *\left(u_{1}+u_{2}\right)$ for some $u_{1} \in W_{1}$, $u_{2} \in W_{2}$. Similarly, $q_{j}=p_{j} *\left(w_{1}+w_{2}\right)$ for some $w_{1} \in W_{1}, w_{2} \in W_{2}$. Let $o_{j}=p_{j} *\left(w_{1}^{\prime}+w_{2}^{\prime}\right)$ $\left(w_{1}^{\prime} \in W_{1}, w_{2}^{\prime} \in W_{2}\right)$ be an arbitrary point on the left coset $p_{j} * W$. Also write $p_{j}=$ $p *\left(x_{1}+\tilde{x}_{1}+x_{2}+\tilde{x}_{2}\right)$ with $x_{i} \in W_{i}, \tilde{x}_{i} \in \tilde{W}_{i}$. Although the $w_{i}$ 's, $x_{i}$ 's and $\tilde{x}_{i}$ 's depend on $r_{j}$, we shall surpress the dependence to simplify the notation.

Next we calculate $d\left(p, p_{j}\right)$ and $d\left(q, o_{j}\right)$. Notice

$$
(-p) * p_{j}=(-p) * p *\left(x_{1}+\tilde{x}_{1}+x_{2}+\tilde{x}_{2}\right)=x_{1}+\tilde{x}_{1}+x_{2}+\tilde{x}_{2} .
$$

So

$$
d\left(p, p_{j}\right)=d\left(o,(-p) * p_{j}\right)=\left|x_{1}\right|+\left|\tilde{x}_{1}\right|+\left|x_{2}\right|^{\frac{1}{2}}+\left|\tilde{x}_{2}\right|^{\frac{1}{2}} .
$$

Using the BCH formula, we obtain:

$$
\begin{aligned}
& (-q) * o_{j} \\
& =(-q) * p_{j} *\left(w_{1}^{\prime}+w_{2}^{\prime}\right) \\
& =\left(-u_{1}-u_{2}\right) *(-p) * p *\left(x_{1}+\tilde{x}_{1}+x_{2}+\tilde{x}_{2}\right) *\left(w_{1}^{\prime}+w_{2}^{\prime}\right) \\
& =\left(-u_{1}-u_{2}\right) *\left(x_{1}+\tilde{x}_{1}+x_{2}+\tilde{x}_{2}\right) *\left(w_{1}^{\prime}+w_{2}^{\prime}\right) \\
& =\left(x_{1}-u_{1}+w_{1}^{\prime}\right)+\tilde{x}_{1}+x_{2}-u_{2}+w_{2}^{\prime}+\tilde{x}_{2}-\frac{1}{2}\left[u_{1}, x_{1}\right]-\frac{1}{2}\left[u_{1}, \tilde{x}_{1}\right]+\frac{1}{2}\left[x_{1}-u_{1}, w_{1}^{\prime}\right]+\frac{1}{2}\left[\tilde{x}_{1}, w_{1}^{\prime}\right] \\
& =\left(x_{1}-u_{1}+w_{1}^{\prime}\right)+\tilde{x}_{1}+P_{2}\left((-q) * o_{j}\right)+\tilde{P}_{2}\left((-q) * o_{j}\right),
\end{aligned}
$$


where

$$
P_{2}\left((-q) * o_{j}\right)=x_{2}-u_{2}+w_{2}^{\prime}-\frac{1}{2}\left[u_{1}, x_{1}\right]+\frac{1}{2}\left[x_{1}-u_{1}, w_{1}^{\prime}\right]+\frac{1}{2} P_{2}\left(\left[\tilde{x}_{1}, u_{1}+w_{1}^{\prime}\right]\right)
$$

and

$$
\tilde{P}_{2}\left((-q) * o_{j}\right)=\tilde{x}_{2}+\frac{1}{2} \tilde{P}_{2}\left(\left[\tilde{x}_{1}, u_{1}+w_{1}^{\prime}\right]\right)
$$

Hence

$$
d\left(q, o_{j}\right)=\left|x_{1}-u_{1}+w_{1}^{\prime}\right|+\left|\tilde{x}_{1}\right|+\left|P_{2}\left((-q) * o_{j}\right)\right|^{\frac{1}{2}}+\left|\tilde{P}_{2}\left((-q) * o_{j}\right)\right|^{\frac{1}{2}} .
$$

Since $q_{j}$ is a point on $p_{j} * W$ nearest to $q$, we have $d\left(q_{j}, q\right) \leq d\left(o_{j}, q\right)$ for any $o_{j} \in p_{j} * W$. By (5.4), we have $d\left(p, p_{j}\right) \leq d\left(q, o_{j}\right) /(101 A)$. In particular, this inequality holds for the point $o_{j}=p_{j} *\left(w_{1}^{\prime}+w_{2}^{\prime}\right)$ where $w_{1}^{\prime}=u_{1}$ and $w_{2}^{\prime}$ is such that $P_{2}\left((-q) * o_{j}\right)=0$. Now using (5.5), (5.7) and (5.8) we obtain:

$$
\begin{aligned}
\left|x_{1}\right|+\left|\tilde{x}_{1}\right|+\left|x_{2}\right|^{\frac{1}{2}}+\left|\tilde{x}_{2}\right|^{\frac{1}{2}} & \leq \frac{1}{101 A}\left(\left|x_{1}\right|+\left|\tilde{x}_{1}\right|+\left|\tilde{x}_{2}+\tilde{P}_{2}\left(\left[\tilde{x}_{1}, u_{1}\right]\right)\right|^{\frac{1}{2}}\right) \\
& \leq \frac{1}{101 A}\left(\left|x_{1}\right|+\left|\tilde{x}_{1}\right|+\left|\tilde{x}_{2}\right|^{\frac{1}{2}}+\left|\tilde{P}_{2}\left(\left[\tilde{x}_{1}, u_{1}\right]\right)\right|^{\frac{1}{2}}\right),
\end{aligned}
$$

which implies

$$
\left|x_{1}\right|+\left|\tilde{x}_{1}\right|+\left|x_{2}\right|^{\frac{1}{2}}+\left|\tilde{x}_{2}\right|^{\frac{1}{2}} \leq \frac{1}{100 A}\left|\tilde{P}_{2}\left(\left[\tilde{x}_{1}, u_{1}\right]\right)\right|^{\frac{1}{2}} .
$$

Next we consider a point $s \in p * W$ of the form $s=p *\left(t u_{1}+u_{2}^{\prime}\right)$, where $u_{2}^{\prime} \in W_{2}$ is arbitrary and $|t| \geq 1$. We claim that

$$
d\left(s, p_{j} * W\right) \geq \frac{\sqrt{|t|}}{2} d\left(q, q_{j}\right)
$$

In the formulas (5.6), (5.7) and (5.8) we replace $u_{1}$ with $t u_{1}, u_{2}$ with $u_{2}^{\prime}$ and $q$ with $s$ to obtain:

$$
\begin{gathered}
P_{2}\left((-s) * o_{j}\right)=x_{2}-u_{2}^{\prime}+w_{2}^{\prime}-\frac{1}{2} t\left[u_{1}, x_{1}\right]+\frac{1}{2}\left[x_{1}-t u_{1}, w_{1}^{\prime}\right]+\frac{1}{2} P_{2}\left(\left[\tilde{x}_{1}, t u_{1}+w_{1}^{\prime}\right]\right), \\
\tilde{P}_{2}\left((-s) * o_{j}\right)=\tilde{x}_{2}+\frac{1}{2} \tilde{P}_{2}\left(\left[\tilde{x}_{1}, t u_{1}+w_{1}^{\prime}\right]\right)
\end{gathered}
$$

and

$$
d\left(s, o_{j}\right)=\left|x_{1}-t u_{1}+w_{1}^{\prime}\right|+\left|\tilde{x}_{1}\right|+\left|P_{2}\left((-s) * o_{j}\right)\right|^{\frac{1}{2}}+\left|\tilde{P}_{2}\left((-s) * o_{j}\right)\right|^{\frac{1}{2}} .
$$

Now for any $o_{j}=p_{j} *\left(w_{1}^{\prime}+w_{2}^{\prime}\right) \in p_{j} * W$, either $\left|w_{1}^{\prime}-t u_{1}\right| \geq \sqrt{|t|}\left|\tilde{P}_{2}\left(\left[\tilde{x}_{1}, u_{1}\right]\right)\right|^{\frac{1}{2}}$ or $\left|w_{1}^{\prime}-t u_{1}\right| \leq \sqrt{|t|}\left|\tilde{P}_{2}\left(\left[\tilde{x}_{1}, u_{1}\right]\right)\right|^{\frac{1}{2}}$. If $\left|w_{1}^{\prime}-t u_{1}\right| \geq \sqrt{|t|}\left|\tilde{P}_{2}\left(\left[\tilde{x}_{1}, u_{1}\right]\right)\right|^{\frac{1}{2}}$, then (5.9) and (5.13) imply

$$
d\left(s, o_{j}\right) \geq\left|x_{1}-t u_{1}+w_{1}^{\prime}\right| \geq\left(\sqrt{|t|}-\frac{1}{100 A}\right)\left|\tilde{P}_{2}\left(\left[\tilde{x}_{1}, u_{1}\right]\right)\right|^{\frac{1}{2}} \geq \frac{\sqrt{|t|}}{2} d\left(q, q_{j}\right) .
$$


If $\left|w_{1}^{\prime}-t u_{1}\right| \leq \sqrt{|t|}\left|\tilde{P}_{2}\left(\left[\tilde{x}_{1}, u_{1}\right]\right)\right|^{\frac{1}{2}}$, then by (15.9)

$$
\begin{aligned}
\left|\left[\tilde{x}_{1}, w_{1}^{\prime}-t u_{1}\right]\right| & \leq A \cdot\left|\tilde{x}_{1}\right| \cdot\left|w_{1}^{\prime}-t u_{1}\right| \\
& \leq A \cdot\left|\tilde{x}_{1}\right| \cdot \sqrt{|t|}\left|\tilde{P}_{2}\left(\left[\tilde{x}_{1}, u_{1}\right]\right)\right|^{\frac{1}{2}} \\
& \leq \frac{1}{100} \sqrt{|t|}\left|\tilde{P}_{2}\left(\left[\tilde{x}_{1}, u_{1}\right]\right)\right|
\end{aligned}
$$

for all sufficiently large $j$. In this case, by (5.13), (5.12) and then (5.9), (5.14)

$$
\begin{aligned}
d\left(s, o_{j}\right) & \geq\left|\tilde{P}_{2}\left((-s) * o_{j}\right)\right|^{\frac{1}{2}} \\
& =\left|\tilde{x}_{2}+\frac{1}{2} \tilde{P}_{2}\left(\left[\tilde{x}_{1}, t u_{1}+w_{1}^{\prime}\right]\right)\right|^{\frac{1}{2}} \\
& =\left|\tilde{x}_{2}+\frac{1}{2} \tilde{P}_{2}\left(\left[\tilde{x}_{1}, w_{1}^{\prime}-t u_{1}\right]\right)+t \tilde{P}_{2}\left(\left[\tilde{x}_{1}, u_{1}\right]\right)\right|^{\frac{1}{2}} \\
& \geq\left|\left(|t|-\frac{1}{(100 A)^{2}}-\frac{1}{2} \cdot \frac{1}{100} \sqrt{|t|}\right) \tilde{P}_{2}\left(\left[\tilde{x}_{1}, u_{1}\right]\right)\right|^{\frac{1}{2}} \\
& \geq \frac{\sqrt{|t|}}{2} d\left(q, q_{j}\right)
\end{aligned}
$$

as $|t| \geq 1$. Hence (5.10) holds.

Set $s^{\prime}=F(s)$. Fix a sequence $r_{j} \rightarrow+0$ such that $l_{F^{-1}}\left(s^{\prime}\right)=\lim _{j} \frac{l_{F^{-1}}\left(s^{\prime}, r_{j}\right)}{r_{j}}$. By (2.2), (5.10) and (5.2)

$$
\begin{aligned}
l_{F^{-1}}\left(s^{\prime}\right) & =\lim _{j} \frac{l_{F^{-1}}\left(s^{\prime}, r_{j}\right)}{r_{j}} \\
& \geq \frac{1}{\eta_{1}(1)} \cdot \limsup _{j} \frac{L_{F^{-1}}\left(s^{\prime}, r_{j}\right)}{r_{j}} \\
& \geq \frac{1}{\eta_{1}(1)} \cdot \limsup _{j} \frac{d\left(s, F^{-1}\left(r_{j}^{2} e * s^{\prime}\right)\right)}{r_{j}} \\
& \geq \frac{\sqrt{|t|}}{2 \eta_{1}(1)} \cdot \limsup _{j} \frac{d\left(q, q_{j}\right)}{r_{j}} \\
& \geq \frac{\sqrt{|t|}}{2\left(\eta_{1}(1)\right)^{2}} \cdot \limsup _{j} \frac{l_{F^{-1}}\left(q^{\prime}, r_{j}\right)}{r_{j}} \\
& \geq \frac{\sqrt{|t|}}{2\left(\eta_{1}(1)\right)^{2}} \cdot l_{F^{-1}}\left(q^{\prime}\right) .
\end{aligned}
$$

The Lemma now follows from (2.1).

Let $\pi_{1}: \mathcal{N}=V_{1} \oplus V_{2} \rightarrow V_{1}$ be the projection onto $V_{1}$. Notice that for each left coset $L=p * W$ of $W$, the projection $\pi_{1}(L)=\pi_{1}(p)+W_{1}$ is an affine subspace of $V_{1}$ parallel to $W_{1}$. By "a hyperplane $H$ in $\pi_{1}(L)$ " we mean an affine subspace $H \subset \pi_{1}(L)$ such that $\operatorname{dim}(H)=\operatorname{dim}\left(\pi_{1}(L)\right)-1$. 
Lemma 5.2. For any two points $p, q \in L$ satisfying $\pi_{1}(p)=\pi_{1}(q)$ we have $l_{F}(p) \leq C_{1}$. $L_{F}(q)$, where $C_{1}=200 A \eta_{1}(1)$.

Proof. We use the notation in the proof of Lemma 5.1. Assume $l_{F}(p)>C_{1} \cdot L_{F}(q)$. Write $q=p *\left(u_{1}+u_{2}\right)$. Then equality (5.9) holds, which implies $u_{1} \neq 0$. Hence $\pi_{1}(p) \neq \pi_{1}(q)$.

Lemma 5.3. Let $L$ be a left coset of $W$ and $p, q \in L$. Suppose $l_{F}(p)>\left(C_{2}\right)^{2 m} L_{F}(q)$, where $m=\operatorname{dim}\left(W_{1}\right)$ and $C_{2}=\max \left\{200 A \eta_{1}(1), 2\left(\eta_{1}(1)\right)^{2}\right\}$. Then there is a hyperplane $H$ in $\pi_{1}(L)$ passing through $\pi_{1}(q)$ and one component $H_{-}$of $\pi_{1}(L) \backslash H$ such that $l_{F}(x) \leq\left(C_{2}\right)^{2 m} L_{F}(q)$ for all $x \in L \cap \pi_{1}^{-1}\left(H_{-}\right)$.

Proof. Let $S$ denote the unit tangent space of $\pi_{1}(L)$ at $\pi_{1}(q)$. We shall define two subsets $G, B$ of $S$. A point $s \in S$ lies in $G$ if $\mathrm{L}_{F}(x) \leq\left(C_{2}\right)^{m} \mathrm{~L}_{F}(q)$ for every $x \in L$ such that $\pi_{1}(x)$ lies in the direction of $s$. A point $s \in S$ lies in $B$ if $\mathrm{l}_{F}(x)>\left(C_{2}\right)^{2 m} \mathrm{~L}_{F}(q)$ for some $x \in L$ such that $\pi_{1}(x)$ lies in the direction of $s$. Clearly $G \cap B=\emptyset$. Let $s_{1} \in S$ be the direction of $\pi_{1}(p)$, and $s_{2} \in S$ the point in $S$ opposite to $s_{1}$. Then $s_{1} \in B$ since $\mathrm{l}_{F}(p)>\left(C_{2}\right)^{2 m} \mathrm{~L}_{F}(q)$. Lemma 5.1 implies $\mathrm{L}_{F}(x) \leq 2\left(\eta_{1}(1)\right)^{2} \mathrm{~L}_{F}(q)$ for any point $x \in L$ such that $\pi_{1}(q) \in \pi_{1}(x) \pi_{1}(p)$. Hence $s_{2} \in G$.

Let $H(B) \subset S$ be the convex hull of $B$ in the sphere $S$. By Caratheodory's theorem for any $y \in H(B)$, there are $m$ points $x_{1}, \cdots, x_{m} \in B$ such that $y$ lies in the spherical simplex $\Delta_{1}$ spanned by $x_{1}, \cdots, x_{m}$. Let $\Delta_{i}$ be the spherical simplex spanned by $x_{i}, \cdots, x_{m}$. Then there are $y_{i} \in \Delta_{i}(1 \leq i \leq m-1)$ with $y_{1}=y$ such that $y_{i} \in x_{i} y_{i+1}$. Since $x_{i} \in B$, there exists a point $p_{i} \in L$ such that $\pi_{1}\left(p_{i}\right)$ lies in the direction of $x_{i}$ with $\mathrm{l}_{F}\left(p_{i}\right)>\left(C_{2}\right)^{2 m} \mathrm{~L}_{F}(q)$. Let $q_{m-1} \in L$ be a point such that $\pi_{1}\left(q_{m-1}\right)$ lies in the direction of $y_{m-1}$ and $\pi_{1}\left(q_{m-1}\right) \in$ $\pi_{1}\left(p_{m-1}\right) \pi_{1}\left(p_{m}\right)$. Inductively, let $q_{i} \in L(1 \leq i \leq m-2)$ be a point such that $\pi_{1}\left(q_{i}\right)$ lies in the direction of $y_{i}$ and $\pi_{1}\left(q_{i}\right) \in \pi_{1}\left(p_{i}\right) \pi_{1}\left(q_{i+1}\right)$.

We claim $\mathrm{L}_{F}(x)>C_{2}^{2 m-1} \mathrm{~L}_{F}(q)$ for every $x \in L$ such that $\pi_{1}(x) \in \pi_{1}\left(p_{m-1}\right) \pi_{1}\left(p_{m}\right)$; in particular, $\mathrm{L}_{F}\left(q_{m-1}\right)>C_{2}^{2 m-1} \mathrm{~L}_{F}(q)$. Suppose the claim does not hold. Then $\mathrm{L}_{F}(x) \leq$ $C_{2}^{2 m-1} \mathrm{~L}_{F}(q)$ for some $x \in L$ satisfying $\pi_{1}(x) \in \pi_{1}\left(p_{m-1}\right) \pi_{1}\left(p_{m}\right)$. Since $l_{F}\left(p_{m-1}\right)>$ $\left(C_{2}\right)^{2 m} \mathrm{~L}_{F}(q)$, we have $\mathrm{l}_{F}\left(p_{m-1}\right)>C_{2} \mathrm{~L}_{F}(x) \geq C_{1} \mathrm{~L}_{F}(x)$. Now Lemma 5.1 implies

$$
\mathrm{L}_{F}\left(p_{m}\right) \leq 2\left(\eta_{1}(1)\right)^{2} \mathrm{~L}_{F}(x) \leq C_{2}^{2 m} \mathrm{~L}_{F}(q),
$$

contradicting $\mathrm{l}_{F}\left(p_{m}\right)>\left(C_{2}\right)^{2 m} \mathrm{~L}_{F}(q)$. By considering $q_{i} \in L$ satisfying $\pi_{1}\left(q_{i}\right) \in \pi_{1}\left(q_{i+1}\right) \pi_{1}\left(p_{i}\right)$ and using Lemma [5.1 one inductively proves that $\mathrm{L}_{F}\left(q_{i}\right)>C_{2}^{m+i} \mathrm{~L}_{F}(q)$. In particular, $\mathrm{L}_{F}\left(q_{1}\right)>C_{2}^{m+1} \mathrm{~L}_{F}(q)$. Since $\pi_{1}\left(q_{1}\right)$ lies in the direction of $y_{1}=y$, we see that $y \notin G$. Since $y \in H(B)$ is arbitrary, we have $H(B) \cap G=\emptyset$.

Now $H(B)$ is a non-empty convex subset of the sphere $S$ and its complement is nonempty. It follows that there is an open hemisphere in its complement. Hence there is an open hemisphere in the complement of $B$. Now the Lemma follows from the definition of $B$.

Lemma 5.4. Suppose $\operatorname{dim}(W) \geq 2$. Then, for any bounded subset $X \subset \pi_{1}(L)$, there exist a subset $E \subset L \cap \pi_{1}^{-1}(X)$ with full measure in $L \cap \pi_{1}^{-1}(X)$ and two constants $M_{1}, M_{2}>0$ such that $L_{F}(x) \geq M_{1}$ and $l_{F}(x) \leq M_{2}$ for all $x \in E$. 
Proof. We first show that there is some $M_{1}>0$ such that $\mathrm{L}_{F}(x) \geq M_{1}$ for all $x \in L \cap \pi_{1}^{-1}(X)$. Suppose there is a sequence of points $x_{i} \in L \cap \pi_{1}^{-1}(X)$ such that $\mathrm{L}_{F}\left(x_{i}\right) \rightarrow 0$. Fix a point $p \in L$ with $\mathrm{l}_{F}(p)>0$. Such a point always exists since $\left.F\right|_{L}: L \rightarrow F(L)$ is quasiconformal and so by Pansu's theorem is a.e. on $L$ Pansu differentiable and the Pansu differential is a.e. on $L$ non-singular. For all sufficiently large $i$ we have $l_{F}(p)>C_{2}^{2 m} \mathrm{~L}_{F}\left(x_{i}\right)$, where $C_{2}$ is the constant in Lemma 5.3. By Lemma 5.3, there is a hyperplane $H_{i}$ of $\pi_{1}(L)$ passing through $\pi_{1}\left(x_{i}\right)$ and a component $H_{i,-}$ of $\pi_{1}(L) \backslash H_{i}$ such that $l_{F}(x) \leq C_{2}^{2 m} \mathrm{~L}_{F}\left(x_{i}\right)$ for all $x \in L \cap \pi_{1}^{-1}\left(H_{i,-}\right)$. Since $X$ is bounded, the sequence $\pi_{1}\left(x_{i}\right)$ is bounded. Hence a subsequence $H_{i_{j},-}$ of the half spaces $H_{i,-}$ converges to an open half space $H_{-}$of $\pi_{1}(L)$. Since every $v \in H_{-}$lies in $H_{i_{j},-}$ for all sufficiently large $j$ and $\mathrm{L}_{F}\left(x_{i}\right) \rightarrow 0$, it follows that $l_{F}(x)=0$ for all $x \in L$ such that $\pi_{1}(x) \in H_{-}$. This means that the quasiconformal map $\left.F\right|_{L}: L \rightarrow F(L)$ has zero differential on non-empty open subsets, contradicting Pansu's theorem.

We next show that $l_{F}$ is essentially bounded from above on $L \cap \pi_{1}^{-1}(X)$. After precomposing and post-composing with left translations we may assume $L=F(L)=W$. Now we can write $L \cap \pi_{1}^{-1}(X)=X \oplus W_{2} \subset W_{1} \oplus W_{2}$. Let $B \subset W_{2}$ be a bounded non-empty open subset of $W_{2}$. Let $X_{1} \subset X$ be the subset of $X$ consisting of all $x \in X$ such that for every $b \in B$ either the Pansu differential $d F(x, b)$ does not exist or $d F(x, b)$ is not a graded isomorphism. By Pansu's theorem and Fubini's theorem, $X_{1}$ has zero measure in $X$. Notice that $E:=L \cap \pi_{1}^{-1}\left(X \backslash X_{1}\right)$ has full measure in $L \cap \pi_{1}^{-1}(X)$. We shall show that $l_{F}$ is bounded from above on $E$. First of all, as a quasisymmetric map, $\left.F\right|_{L}: L \rightarrow F(L)$ maps bounded sets to bounded sets. So $F(X \oplus B)$ is bounded. Now the first paragraph applied to $F^{-1}$ implies that there is a positive lower bound for $\mathrm{L}_{F^{-1}}$ on $F(X \oplus B)$. By (2.1) there is some $M_{1}>0$ such that $l_{F}\left(q_{1}\right) \leq M_{1}$ for any $q_{1} \in X \oplus B$. Now let $q \in E$ be arbitrary. Then by the definition of $E$ there is some $q_{1} \in X \oplus B$ such that $\pi_{1}(q)=\pi_{1}\left(q_{1}\right)$ and the Pansu differential $d F\left(q_{1}\right)$ is a graded isomorphism. By Lemma 5.2 and $(2.2)$ we have

$$
l_{F}(q) \leq C_{1} L_{F}\left(q_{1}\right) \leq C_{1} \eta(1) l_{F}\left(q_{1}\right) \leq C_{1} \eta(1) M_{1} .
$$

Lemma 5.5. For each left coset $L$ of $W$, there is some constant $C_{L}>0$ such that $\left.F\right|_{L}$ is $a\left(C_{2}^{2 m+3}, C_{L}\right)$-quasi-similarity, where $m=\operatorname{dim}(W)$ and $C_{2}$ is the constant in Lemma 5.3 .

Proof. First consider the case when $\operatorname{dim}(W)=1$. By Pansu's differentiability theorem and the theorem in [BKR] on absolute continuity on almost all curves, we conclude that for a.e. left coset $L$ the following hold: $F$ is absolutely continuous on $L$, at a.e. point $q \in L$, the Pansu differential $d F(q)$ is a graded isomorphism. Fix any such $L$. We first observe that to show $\left.F\right|_{L}$ is a quasi-similarity, it suffices to show that $l_{F}(p) \leq C_{1} \cdot \mathrm{L}_{F}(q)$ for any $p, q \in L$, where $C_{1}$ is the constant in Lemma 5.1. Suppose there are two points $p, q \in L$ such that $\mathrm{l}_{F}(p)>C_{1} \cdot \mathrm{L}_{F}(q)$. By Lemma 5.1, $\mathrm{L}_{F}(x) \rightarrow 0$ as $d(p, x) \rightarrow \infty(x \in L)$. By (2.1) we have $\mathrm{L}_{F^{-1}}(y) \geq \mathrm{l}_{F^{-1}}(y) \rightarrow \infty$ as $d(y, F(p)) \rightarrow \infty(y \in F(L))$. However, $\mathrm{l}_{F}(p)>C_{1} \mathrm{~L}_{F}(q)$ implies $\mathrm{l}_{F^{-1}}(F(q))>C_{1} \cdot \mathrm{L}_{F^{-1}}(F(p))$. Applying Lemma 5.1 to $F^{-1}$ we obtain $\mathrm{L}_{F^{-1}}(y) \rightarrow 0$, which is a contradiction. Hence for a.e. left coset $L$ of $W,\left.F\right|_{L}$ is a $\left(C_{2}^{2}, C_{L}\right)$-quasi-similarity for some $C_{L}$. By continuity, this holds for every left coset of $W$.

From now on we assume $\operatorname{dim}(W) \geq 2$. In this case, both $\left.F\right|_{L}$ and $\left.F^{-1}\right|_{F(L)}$ have the following properties: (1) absolutely continuous, (2) Pansu differentiable a.e. and the Pansu 
differential is a.e. a graded isomorphism, (3) absolutely continuous on almost all curves. It follows that to show $\left.F\right|_{L}$ is a $\left(C_{2}^{2 m+3}, C_{L}\right)$ quasi-similarity for some $C_{L}>0$, it suffices to show that there is a set of full measure $E \subset L$ such that $l_{F}(x) \leq \eta(1) \eta_{1}(1) C_{2}^{2 m+1} \mathrm{~L}_{F}(y)$ for all $x, y \in E$. We shall prove this by contradiction. So suppose the above statement is not true. Then in particular there are two points $p, q \in L$ such that $\mathrm{l}_{F}(p)>\eta(1) \eta_{1}(1) C_{2}^{2 m+1} \mathrm{~L}_{F}(q)$. By Pansu's theorem, we may assume $l_{F}(p)<\infty$.

We first observe that it suffices to show that there is a constant $b_{0} \geq 1$ such that $\mathrm{l}_{F}(x) \leq b_{0}$ for all $x$ in a full measure subset of $L$ : the condition $\mathrm{l}_{F}(p)>\eta(1) \eta_{1}(1) C_{2}^{2 m+1} \mathrm{~L}_{F}(q)$ implies that $\mathrm{l}_{F^{-1}}(F(q))>\eta(1) \eta_{1}(1) C_{2}^{2 m+1} \mathrm{~L}_{F^{-1}}(F(p))$. Then Lemma 5.1 implies that there is some $y_{0} \in F(L)$ such that

$$
\mathrm{L}_{F^{-1}}\left(y_{0}\right)<\frac{1}{b_{0} C_{2}^{2 m} \eta_{1}(1)} \min \left\{1,1_{F^{-1}}(F(q))\right\} .
$$

By Lemma 5.3, there is a hyperplane $H^{\prime}$ in $\pi_{1}(F(L))$ passing trough $\pi_{1}\left(y_{0}\right)$ and a component $H_{-}^{\prime}$ of $\pi_{1}(F(L)) \backslash H^{\prime}$ such that $l_{F^{-1}}(y)<\frac{1}{b_{0} \eta_{1}(1)}$ for all $y \in F(L) \cap \pi_{1}^{-1}\left(H_{-}^{\prime}\right)$. Since $F^{-1}$ is Pansu differentiable a.e., $\mathrm{L}_{F^{-1}}(y)<\frac{1}{b_{0}}$ for a.e. $y \in F(L) \cap \pi_{1}^{-1}\left(H_{-}^{\prime}\right)$. It follows that $\mathrm{l}_{F}(x)>b_{0}$ for a.e. $x \in L \cap F^{-1}\left(\pi_{1}^{-1}\left(H_{-}^{\prime}\right)\right)$, contradicting the assumption that $\mathrm{l}_{F}(x) \leq b_{0}$ for all $x$ in a full measure subset of $L$.

Since we assume there are two points $p, q \in L$ such that $l_{F}(p)>\eta(1) \eta_{1}(1) C_{2}^{2 m+1} \mathrm{~L}_{F}(q)$, by Lemma 5.3, there is a hyperplane $H_{1}$ in $\pi_{1}(L)$ passing through $\pi_{1}(q)$ and one component $H_{1,-}$ of $\pi_{1}(L) \backslash H_{1}$ such that

$$
\mathrm{l}_{F}(x) \leq\left(C_{2}\right)^{2 m} \mathrm{~L}_{F}(q)<\frac{1}{\eta(1) \eta_{1}(1) C_{2}} \mathrm{l}_{F}(p)
$$

for all $x \in L \cap \pi_{1}^{-1}\left(H_{1,-}\right)$. Now Pansu's theorem and the quasisymmetry condition on $F$ imply $\mathrm{L}_{F}(x)<\frac{1}{\eta_{1}(1) C_{2}} l_{F}(p)$ for a.e. $x \in L \cap \pi_{1}^{-1}\left(H_{1,-}\right)$. Let $\tau: \pi_{1}(L) \rightarrow \pi_{1}(L)$ be the geodesic symmetry about $\pi_{1}(p)$, that is, for any $v \in \pi_{1}(L), \tau(v)$ is such that $\pi_{1}(p)$ is the midpoint of $v \tau(v)$. Now Lemma 5.1 implies that for a.e. $y \in L \cap \pi_{1}^{-1}\left(\tau\left(H_{1,-}\right)\right)$ we have

$$
\mathrm{L}_{F}(y) \leq 2\left(\eta_{1}(1)\right)^{2} \mathrm{~L}_{F}(\tau(y)) \leq \frac{2 \eta_{1}(1)}{C_{2}} l_{F}(p) .
$$

Hence $L_{F}(x)$ is essentially bounded on $L \cap\left(\pi_{1}^{-1}\left(H_{1,-}\right) \cup \pi_{1}^{-1}\left(\tau\left(H_{1,-}\right)\right)\right)$. If $\pi_{1}(p) \in H_{1}$, then we are done since now $\mathrm{L}_{F}(x)$ (hence $l_{F}(x)$ ) is bounded on the full measure subset $L \cap\left(\pi_{1}^{-1}\left(H_{1,-}\right) \cup \pi_{1}^{-1}\left(\tau\left(H_{1,-}\right)\right)\right)$ of $L$. So we assume $\pi_{1}(p) \notin H_{1}$. Let $B_{1} \subset \pi_{1}(L)$ be the part of a cylinder in $\pi_{1}(L)$ with center line passing through $\pi_{1}(p)$, perpendicular to the hyperplane $H_{1}$ and bounded between $H_{1}$ and $\tau\left(H_{1}\right)$. Then $B_{1}$ is bounded. By Lemma 5.4 there are numbers $M_{1}, M_{2}>0$ and a set $E_{1} \subset L \cap \pi_{1}^{-1}\left(B_{1}\right)$ with full measure in $L \cap \pi_{1}^{-1}\left(B_{1}\right)$ such that $\mathrm{L}_{F}(x) \geq M_{1}$ and $l_{F}(x) \leq M_{2}$ for every $x \in E_{1}$.

Since we assume $l_{F}(x)$ is not essentially bounded, there is some hyperplane $\tilde{H}_{1}$ of $\pi_{1}(L)$ parallel to $H_{1}$ such that (1) $F$ is Pansu differentiable at some $q_{1} \in E_{1} \cap \pi_{1}^{-1}\left(B_{1} \cap \tilde{H}_{1}\right)$; (2) there is some $p_{1} \in \pi_{1}^{-1}\left(\tilde{H}_{1}\right)$ with $C_{2}^{2 m+1} \cdot(\eta(1))^{2} M_{2}<1_{F}\left(p_{1}\right)<\infty$. Since $F$ is Pansu differentiable at $q_{1}$, we have $\mathrm{L}_{F}\left(q_{1}\right) \leq \eta(1) \mathrm{l}_{F}\left(q_{1}\right) \leq \eta(1) M_{2}$. So $\mathrm{l}_{F}\left(p_{1}\right)>C_{2}^{2 m+1} \eta(1) \cdot \mathrm{L}_{F}\left(q_{1}\right)$. Now, as indicated above, Lemma 5.3 and Lemma 5.1 imply that there is a hyperplane $H_{2}$ 
of $\pi_{1}(L)$ passing through $\pi_{1}\left(q_{1}\right)$ and a component $H_{2,-}$ of $\pi_{1}(L) \backslash H_{2}$ such that $\mathrm{L}_{F}(x)$ is essentially bounded on $\pi_{1}^{-1}\left(H_{2,-}\right)$ and $\pi_{1}^{-1}\left(\tau_{1}\left(H_{2,-}\right)\right)$, where $\tau_{1}$ is the geodesic symmetry in $\pi_{1}(L)$ about $\pi_{1}\left(p_{1}\right)$. If $\pi_{1}\left(p_{1}\right) \in H_{2}$, then we are done as indicated above. So we assume $\pi_{1}\left(p_{1}\right) \notin H_{2}$. In this case, $H_{1}$ and $H_{2}$ are not parallel. We proceed inductively and eventually find $m$ hyperplanes $H_{1}, H_{2}, \cdots, H_{m}$, half spaces $H_{i,-}$ and points $p_{0}:=$ $p, p_{1}, \cdots, p_{m-1}$ with the following properties:

(1) $\mathrm{L}_{F}(x)$ (hence $l_{F}(x)$ ) is essentially bounded on $\pi_{1}^{-1}(Q)$, where $Q$ is the union $Q:=$ $\cup_{i} H_{i,-} \cup \cup_{i} \tau_{i-1}\left(H_{i,-}\right)$, where $\tau_{i-1}$ is the geodesic symmetry about the point $\pi_{1}\left(p_{i-1}\right)$;

(2) The complement of $Q$ in $\pi_{1}(L)$ is compact.

By Lemma 5.4, $1_{F}(x)$ is essentially bounded on $L \backslash \pi_{1}^{-1}(Q)$. It follows that $\mathrm{l}_{F}(x)$ is essentially bounded on $L$, and we are done.

Now Theorem 1.1 follows from Lemma 5.5 and the proof of Theorem 3.2 .

\section{$6 \quad$ Examples}

In this Section we apply our results to 3 examples.

We call a Carnot algebra quasisymmetrically rigid if the corresponding Carnot group is quasisymmetrically rigid. All the examples here are quasisymmetrically rigid. The first example satisfies $r_{1}(\mathcal{N})=1$, and so is non-rigid by [O]. Similarly for the Carnot algebras in the second example as soon as $r_{1}(\mathcal{N})=1$ for the factor $\mathcal{N}$. The last example is rigid since $r_{1}(\tilde{\mathcal{N}})=5$.

First Example. Let $\mathcal{N}=V_{1} \oplus V_{2} \oplus V_{3}$ be a 3-step Carnot algebra defined as follows. The

first layer $V_{1}$ has basis $\left\{X, Y, X^{\prime}, Y^{\prime}, X_{1}, X_{2}\right\}$, the second layer $V_{2}$ has basis $\left\{Z, X_{1,2}\right\}$, and the third layer $V_{3}$ has basis $\left\{X_{1,2,1}, X_{1,2,2}\right\}$. The only non-trivial bracket relations are

$$
\begin{gathered}
{[X, Y]=\left[X^{\prime}, Y^{\prime}\right]=Z,\left[X_{1}, X_{2}\right]=X_{1,2},\left[X, X_{2}\right]=\left[Y, X_{2}\right]=X_{1,2},} \\
{\left[X_{1,2}, X_{1}\right]=\left[X_{1,2}, X\right]=\left[X_{1,2}, Y\right]=X_{1,2,1},\left[X_{1,2}, X_{2}\right]=X_{1,2,2} .}
\end{gathered}
$$

Let $0 \neq v \in V_{1}$. Write $v=a X+b Y+a_{1} X_{1}+b_{1} X_{2}+a^{\prime} X^{\prime}+b^{\prime} Y^{\prime}$. A direct calculation shows

$$
\begin{gathered}
{[v, X]=-b Z-b_{1} X_{1,2},} \\
{[v, Y]=a Z-b_{1} X_{1,2},} \\
{\left[v, X_{1}\right]=-b_{1} X_{1,2},} \\
{\left[v, X_{2}\right]=\left(a+b+a_{1}\right) X_{1,2},} \\
{\left[v, X^{\prime}\right]=-b^{\prime} Z,} \\
{\left[v, Y^{\prime}\right]=a^{\prime} Z,} \\
{[v, Z]=0,} \\
{\left[v, X_{1,2}\right]=-b_{1} X_{1,2,2}-\left(a+b+a_{1}\right) X_{1,2,1} .}
\end{gathered}
$$


It is easy to check that $\operatorname{rank}(v) \geq 1$ and $\operatorname{rank}(v)=1$ if and only if $b_{1}=0$ and $a+b+a_{1}=0$. Hence $W_{1}=\left\{a X+b Y+a_{1} X_{1}+a^{\prime} X^{\prime}+b^{\prime} Y^{\prime}: a+b+a_{1}=0\right\}$ is a non-trivial proper subspace of $V_{1}$ invariant under the action of $\operatorname{Aut}_{g}(\mathcal{N})$. Notice that $X-Y, X-X_{1}, X^{\prime}, Y^{\prime}$ form a basis for $W_{1}$ and the subalgebra $\left\langle W_{1}\right\rangle=W_{1} \oplus \mathbb{R} Z$ is isomorphic to the second Heisenberg algebra. Since $N$ is 3-step, one can not apply Theorem 1.1. Also $<W_{1}>$ is a Heisenberg algebra and so Theorem 1.3 is not applicable (there exist non-biLipschitz quasiconformal maps on the Heisenberg groups [B]). However, (6.1) implies $\left[X_{1}, W_{1}\right]=0$. Since $X_{1} \notin W_{1}$, by Theorem $1.2, \mathcal{N}$ is quasisymmetrically rigid.

Second Example. Let $\mathcal{N}=V_{1} \oplus V_{2}, \mathcal{N}^{\prime}=V_{1}^{\prime} \oplus V_{2}^{\prime}$ be 2-step Carnot algebras. If $r_{1}(\mathcal{N}) \neq r_{1}\left(\mathcal{N}^{\prime}\right)$, then the discussion in Section 4.2 and Theorem 1.1 show that the direct product $\mathcal{N} \oplus \mathcal{N}^{\prime}$ and all their central products are quasisymmetrically rigid. If $r_{1}(\mathcal{N}) \geq 1$ and $r_{1}\left(\mathcal{N}^{\prime}\right) \geq 1$, then Lemma 4.2 and Theorem 1.1 imply that all level one products of $\mathcal{N}$ and $\mathcal{N}^{\prime}$ are quasisymmetrically rigid.

Third Example. Next we construct a Carnot algebra from the model Filiform algebra $\mathcal{F}^{4}$ and the free nilpoptent Lie algebra $\mathcal{F}_{2,6}$ (2-step with 6 generators). Recall that $\mathcal{F}^{4}=$ $V_{1} \oplus V_{2} \oplus V_{3} \oplus V_{4}$ with $V_{1}$ spanned by $e_{1}, e_{2}, V_{2}$ spanned by $e_{3}, V_{3}$ spanned by $e_{4}$ and $V_{4}$ spanned by $e_{5}$. The only non-trivial bracket relations are $\left[e_{1}, e_{i}\right]=e_{i+1}$ for $i=2,3,4$. The free nilpotent Lie algebra $\mathcal{F}_{2,6}=V_{1}^{\prime} \oplus V_{2}^{\prime}$, where $V_{1}^{\prime}$ is spanned by $\left\{X_{i}: 1 \leq i \leq 6\right\}$ and $V_{2}^{\prime}$ is spanned by $\left\{X_{i j}: 1 \leq i<j \leq 6\right\}$. The only non-trivial bracket relations are $\left[X_{i}, X_{j}\right]=X_{i j}$ for $i<j$. The Carnot algebra $\mathcal{N}$ is obtained from $\mathcal{F}^{4} \oplus \mathcal{F}_{2,6}$ by adding the following bracket relations:

$$
\begin{aligned}
& {\left[e_{2}, X_{2}\right]=X_{13},\left[e_{2}, X_{3}\right]=X_{24},\left[e_{2}, X_{4}\right]=X_{35},} \\
& {\left[e_{2}, X_{6}\right]=X_{15}, \quad\left[e_{1}, X_{1}\right]=X_{26},\left[e_{1}, X_{5}\right]=X_{46} .}
\end{aligned}
$$

Now it is easy to check that $\operatorname{rank}\left(e_{1}\right)=\operatorname{rank}\left(e_{2}\right)=5$. Furthermore, a tedious calculation shows $\operatorname{rank}(v) \geq 6$ for $v \in\left(V_{1} \oplus V_{1}^{\prime}\right) \backslash V_{1}$. Hence $W_{1}:=V_{1}$ is a non-trivial proper subspace of $V_{1} \oplus V_{1}^{\prime}$ invariant under the action of $\operatorname{Aut}_{g}(\mathcal{N})$ and $\left\langle W_{1}\right\rangle=\mathcal{F}^{4}$. It was proved in [X2] that $\mathcal{F}^{4}$ is quasisymmetrically rigid. Now Theorem 1.3 implies that $\mathcal{N}$ is also quasisymmetrically rigid.

The group $N$ is 4-step, so Theorem 1.1 does not apply. It also does not satisfy the condition in Theorem [1.2. Indeed, let $X=a_{1} e_{1}+a_{2} e_{2}+\sum_{i=1}^{6} b_{i} X_{i}$. Now $\left[e_{1}, X\right]=a_{2} e_{3}+$ $b_{1} X_{26}+b_{5} X_{46}$ and $\left[e_{2}, X\right]=-a_{1} e_{3}+b_{2} X_{13}+b_{3} X_{24}+b_{4} X_{35}+b_{6} X_{15}$. So $\left[X, W_{1}\right] \subset<W_{1}>$ if and only if $X \in W_{1}$.

\section{References}

[B] Z. Balogh, Hausdorff dimension distribution of quasiconformal mappings on the Heisenberg group, J. Anal. Math. 83 (2001), 289-312.

[BKR] Z. Balogh, P. Koskela and S. Rogovin, Absolute continuity of quasiconformal mappings on curves, Geom. Funct. Anal. 17 (2007), no. 3, 645-664.

[BR] A. Bellaiche, J. J. Risler, Sub-Riemannian Geometry, Progress in Mathematics 144, Basel 1996. 
[CC] L. Capogna, M. Cowling, Conformality and Q-harmonicity in Carnot groups, Duke Math. J. 135 (2006), no. 3, 455-479.

[CG] L. Corwin, F. Greenleaf, Representations of nilpotent Lie groups and their applications, Part I. Basic theory and examples, Cambridge Studies in Advanced Mathematics, 18. Cambridge University Press, Cambridge, 1990.

[H] E. Heintze, On homogeneous manifolds of negative curvature, Math. Ann. 211 (1974), $23-34$.

[HK] J. Heinonen, P. Koskela, Quasiconformal maps in metric spaces with controlled geometry, Acta Math. 181 (1998), no. 1, 1-61.

[O] A. Ottazzi, A sufficient condition for nonrigidity of Carnot groups, Math. Z. 259 (2008), no. 3, 617-629.

[OW] A. Ottazzi, B. Warhurst, Contact and 1-quasiconformal maps on Carnot groups, J. Lie Theory 21 (2011), no. 4, 787-811.

[P] P. Pansu, Metriques de Carnot-Caratheodory et quasiisometries des espaces symetriques de rang un, Ann. of Math. (2) 129 (1989), no. 1, 1-60.

[R] H. M. Reimann, Rigidity of H-type groups, Math. Z. 237 (2001), no. 4, 697-725.

[SX] N. Shanmugalingam, X. Xie, A Rigidity Property of Some Negatively Curved Solvable Lie Groups, Comment. Math. Helv. 87 (2012), no. 4, 805-823.

[V] J. Väisälä, The free quasiworld. Freely quasiconformal and related maps in Banach spaces, Quasiconformal geometry and dynamics, Banach Center Publ. (Lublin) 48 (1996), 55-118.

[X1] X. Xie, Quasiconformal maps on non-rigid Carnot groups, in preparation.

[X2] X. Xie, Quasiconformal maps on model Filiform groups, preprint.

[X3] X. Xie, Quasisymmetric maps on reducible Carnot groups, to appear in Pacific Journal of Mathematics.

Address:

Xiangdong Xie: Dept. of Mathematics and Statistics, Bowling Green State University, Bowling Green, OH, U.S.A. E-mail: xiex@bgsu.edu 